9

10

11

\section{Area-specific synapse structure in branched axons reveals a subcellular level of complexity in thalamocortical networks}

4 Javier Rodriguez-Moreno ${ }^{1}$, Cesar Porrero ${ }^{1}$, Astrid Rollenhagen ${ }^{2}$, Mario Rubio-Teves ${ }^{1}$,

Diana Casas-Torremocha ${ }^{1}$, Lidia Alonso-Nanclares ${ }^{3}$, Rachida Yakoubi ${ }^{2}$, Andrea

Santuy $^{3}$, Angel Merchan-Pérez ${ }^{3,5,6}$, Javier DeFelipe ${ }^{3,4,5}$, Joachim HR Lübke ${ }^{2,7,8+}$ and

Francisco Clasca $^{1+*}$

(1) Department of Anatomy \& Neuroscience, School of Medicine, Autónoma de Madrid University, 28029 Madrid, Spain;

1 (2) Institute of Neuroscience and Medicine INM-10, Research Centre Jülich GmbH, 52425 Jülich, Germany;

3 (3) Laboratorio Cajal de Circuitos Corticales, Centro de Tecnología Biomédica, 14 Universidad Politécnica de Madrid, Pozuelo de Alarcón, Madrid, Spain;

5 (4) Instituto Cajal, Consejo Superior de Investigaciones Científicas, 28002, Madrid, 16 Spain;

(5) CIBERNED, Centro de Investigación Biomédica en Red de Enfermedades Neurodegenerativas;

9 (6) Departamento de Arquitectura y Tecnología de Sistemas Informáticos, Universidad 0 Politécnica de Madrid. Boadilla del Monte, 28660, Madrid, Spain;

1 (7) Department of Psychiatry, Psychotherapy and Psychosomatics, RWTH Aachen University, Germany;

(8) JARA-Brain Medicine, Jülich-Aachen, Germany.

+ both authors share senior authorship

*Author for correspondence Prof. Francisco Clascá Dept. of Anatomy \& Graduate Program in Neuroscience Autónoma de Madrid University, School of Medicine Calle Arzobispo Morcillo 4, Madrid, SPAIN 28029 Tel. +34 - 666990337 e-mail: francisco.clasca@uam.es https://orcid.org/0000-0003-0718-1337 


\section{Abstract}

37

38 Thalamocortical Posterior nucleus (Po) axons innervating the somatosensory (S1) and

39 motor (MC) vibrissal cortices are key links in the brain neuronal network that allows

40 rodents to explore the environment whisking with their motile vibrissae. Here, using

41 high-end 3D electron microscopy, we demonstrate massive differences between MC vs.

42 S1 Po synapses in a) bouton and active zone size; b) neurotransmitter vesicle pool size;

43 c) mitochondria distribution near synapses; and d) proportion of non-spinous dendrite

44 contacts. These differences are as large, or bigger, than those between Po and

45 ventroposterior thalamic nucleus synapses in S1. Moreover, using single-axon

46 transfection labeling, we show that the structure of boutons in the MC vs. S1 branches

47 of individual Po axons is different. These structural differences parallel striking,

48 recently-discovered divergences in functional efficacy and plasticity between S1 and

49 MC Po synapses, and overall reveal a new, subcellular level of thalamocortical circuit 50 complexity, unaccounted for in current models. 


\section{Introduction}

53

54 Rodents explore their environment by rhythmically "whisking" with their motile facial vibrissae. Time-dependent correlations between motor commands and vibrissal follicle mechanoreceptor signals are used for inferring object position and texture. Such computations are carried out in multilevel, closed-loop neuronal networks encompassing the brainstem, thalamus and neocortex (reviewed in ${ }^{1}$ ).

Two thalamocortical pathways lay at the core of these loops: Ventral Posteromedial thalamic nucleus (VPM) axons arborizing focally on L4 in the vibrissal "barrel" domain of the primary somatosensory cortex (S1); and Posterior thalamic nucleus (Po) axons arborizing mainly in L5a but also L1 of $\mathrm{S}^{2}{ }^{2 ;}$. Importantly, Po axons may target, in addition, the motor cortex (MC) middle layers ${ }^{3,4,5}$ (L5-L3). The VPM axons relay time-locked mechanoreceptive single-whisker trigeminal signals, crucial for computing object location. In turn, Po axons convey information mainly about timing/amplitude differences between ongoing cortical outputs and multi-whisker sensory signals, which may be important for computing whisker position ${ }^{1,6,7}$. Po cells are primarily driven by heavy and highly effective L5 cortico-thalamic inputs, while ascending trigeminal inputs to Po are sparser and largely modulatory in character ${ }^{7,8}$.

Activation of S1-L4 VPM synapses elicits large currents in cortical neurons $9 ; 10$ and drives their firing with low failure rates ${ }^{11,12}$. VPM synapses are driven only by ionotropic receptors, depress rapidly upon repetitive stimulation ${ }^{9}$ and, after an early postnatal period, show considerable resistance to sensory experience-dependent changes times, and elicit smaller currents ${ }^{10,14}$. The Po S1 synapses involve both ionotropic and metabotropic glutamate conductances, show paired-pulse facilitation ${ }^{10,15}$ and rapidly increase their efficacy in response to learned reward even in the adult ${ }^{13}$. Recent data about the Po MC synapses indicate that, surprisingly, they elicit large currents, depress upon repetitive activation ${ }^{5,16}$ and involve only ionotropic receptors ${ }^{3}$.

In cortical synapses, bouton and mitochondrial volume, synaptic vesicle pool size, and postsynaptic density (PSD) complexity and surface area directly correlate with increased neurotransmitter release probability and synaptic strength ${ }^{17,18 ; 19}$.

83 Furthermore, the PSD surface area is proportional to the number of postsynaptic

84 receptors $20,21,22,23$. Thus, given the markedly divergent effect observed in S1 vs. MC

85 Po synapses, and recent light microscope evidence that Po S1 axon varicosities are 
86 relatively small ${ }^{3}$ we aimed to determine whether Po synapses in MC and S1 were

87 different in their ultrastructural composition. Moreover, as studies in rat have reported

88 that MC and S1 can be simultaneously targeted by branches of the same individual Po

89 axons ${ }^{4,24}$, we also set out to elucidate if structural differences occur between boutons

90 on separate branches of the same Po cell axon.

91 To visualize Po axons and measure identified Po synapses in S1 and MC, we

92 combined single-cell and bulk axonal labeling with light and high-resolution, fine-scale

93 3D-electron microscopy (serial sectioning Transmission Electron Microscopy, ssTEM;

94 and Focused Ion Beam Milling Scanning Electron Microscopy; FIB-SEM). In addition,

95 as Po and VPM synapses have been recently shown to elicit marked different effects on

96 S1 neurons, we re-analyzed our own dataset on VPM S1 synapses ${ }^{25}$ and compared it

97 with the Po synapse data.

98 Here, we demonstrate that axonal varicosities of Po axons in $\mathrm{MC}$ are much larger

99 than those of the same axons in S1, and that their presynaptic structure and postsynaptic

100 relationships in each cortical area are strikingly different. In addition, we report

101 similarly sharp differences between Po and VPM axon synapses in S1. Along with

102 recent parallel functional observations ${ }^{3,16}$ differences in synapse structure both between

103 axons originated in diverse thalamic nuclei, and between the branches of the same

104 individual axons targeting separate cortical areas/layers, call into question current

105 models of thalamocortical interaction. 


\section{Results}

107

Light microscopic visualization of Po axon terminals in S1 and in MC

To selectively label a sizable population of thalamocortical Po axons, $10 \mathrm{kDa}$ lysinefixable biotinylated dextranamine (BDA) was iontophoretically delivered into Po. Only experiments in which the BDA deposit was restricted to the Po nucleus were analyzed (Fig. 1a-b; Supplementary Fig. SM1). In S1, two distinct bands of axonal arborizations were labeled: one in L5a and the other in upper half of L1 (Fig. 1c). In MC, labeled Po axonal arborizations formed a single band from upper L5a to lower L3 (Fig. 1d). The Golgi-like axon staining revealed frequent varicosities of variable size (Fig. 1e-g).

\section{SSTEM and FIB-SEM analysis of labeled thalamocortical Po axons}

120 Electron microscopic samples were taken from sections adjacent to those containing the heaviest anterograde labeling in S1 or MC (see Methods; Supplementary Fig. SM2). $\mathrm{S} 1$, samples were taken from L1 and L5a, which were readily delineated cytoarchitecturally. However, the cytoarchitectonic definition of MC middle layers is ambiguous; thus, our MC samples included L4 and adjacent deep parts of L3; we refer to this neuropil as "MC-L4/3".

A total of 220 Po synaptic boutons and their respective target structures were reconstructed and analyzed with ssTEM or FIB-SEM: 80 in S1-L5a, 71 in S1-L1 and 69 in MC-L4/3. The two serial 3D EM techniques yielded in consistent results. The ssTEM analysis allowed a high-resolution 3D-volume reconstruction of the overall geometry of synaptic complexes including the subsequent quantification of PSDs surface area and vesicle pool size, however, it sampled only axon varicosities (putative boutons), and a smaller neuropil volume, which not always allowed a full visualization of the spine neck. In turn, FIB-SEM analysis allowed the complete visualization of postsynaptic elements and inter-bouton axonal segments. For efficiency, the FIB-SEM image acquisition was made with a slightly lower resolution than the ssTEM images; for this reason, fine details such as the individual synaptic vesicles could be only unambiguously resolved and counted in the ssTEM samples. 
Ultrastructural features of Po synaptic boutons in the vibrissal regions of S1 and MC

A total of 192 axon boutons, most, but not all of them containing at least one synaptic boutons from S1-L5a, 67 boutons from S1-L1 and 51 boutons from MC-L4/3 (Table 1). Labeled boutons were identified by their electron-dense DAB reaction product in their cytoplasm and by their tapering into the adjacent axonal segments. A second criterion was the presence of a postsynaptic density (PSD) in consecutive serial sections indicative for a synaptic contact. The sampling of labeled boutons in SsTEM was essentially random, as any labeled bouton that could be followed from its beginning to its end within a given series of ultrathin sections was reconstructed and quantified. In FIB-SEM image stacks, all the labeled axonal segments, varicose or not, contained in the stack volume were analyzed.

Synaptic contacts between a labeled Po axon and its postsynaptic target were identified by the presence of distinct, parallel pre- and postsynaptic membranes at the synaptic apposition zone, separated by a synaptic cleft and an electron-dense band adherent to the cytoplasmic surface of the postsynaptic membrane (PSD). This corresponds to asymmetric synapses, regarded as excitatory and thus glutamatergic ${ }^{26}$. Mitochondria and synaptic vesicles were clearly visible and, in the boutons less heavily-stained, it was even possible to identify and count with confidence all the synaptic vesicles.

In the three cortex regions studied, the large majority of Po boutons were monosynaptic. About $30 \%$ of boutons in MC-L4/3 and $10 \%$ in S1 (L5a and L1) simultaneously innervated two target structures (Fig. 2d, 2f, Table 1). We did not observe any Po bouton with three or more synapses, which is a frequent finding in S1-L4 VPM boutons 25 (Table 1).

172 Most Po boutons (88-92\%, Table 1) contained one or several mitochondria of 173 different shape and size (Fig. 2 and 3). Mitochondria contributed substantially to the 
174 bouton volume (25\% in S1-L5a, 16\% in S1-L1 and 20.5\% in MC-L4/3). Remarkably, in

175 both S1-L1 and S1-L5a, a significant number (7\%) of the Po axonal varicosities that

176 contained a mitochondrion and synaptic vesicles, lacked any evident synaptic contact.

177 Such "non-synaptic boutons" were also observed in MC-L4/3 Po axons but less

178 frequent (2\%), but never in the VPM S1-L4 axons (Table 1; see also ${ }^{27}$ ).

179

180

181

182

183

184

185

186

187

188

189

190

191

192

193

194

195

196

197

198

199

200

201

202

203

204

205

206

207

Insert Figure 4 about here

\section{Synaptic structural features specifically revealed by FIB-SEM analysis}

Our FIB-SEM analysis is based on sampling 2,435 $\mu \mathrm{m}^{3}$ of cortical neuropil. At high magnification FIB-SEM, the axonal BDA axonal segments were few and widely scattered. Thus, despite taking our FIB-SEM samples from zones that under light microscopy appeared heavily labeled (Fig. 1), many of the volumes examined with FIBSEM actually contained inside few or no labeled Po axonal segments. Nevertheless, twenty-six Po axonal segments totaling $\sim 283 \mu \mathrm{m}$ of axonal length were reconstructed. Of these, $108 \mu \mathrm{m}$ were measured from axonal branches in S1-L5a (0.16 synapses/ $\mu \mathrm{m}$ of total axon length); $74 \mu \mathrm{m}$ from S1-L1 (0.05 synapses/ $\mu \mathrm{m}$ of total axon length); and 101 $\mu \mathrm{m}$ from MC-L4/3 (0.14 synapses/ $\mu \mathrm{m}$ of total axon length; Table 1, Fig. 4, Supplementary Fig. SM3). Varicosities were defined as any swelling on the axon exceeding by more than $50 \%$ the typical variation of the adjacent axonal segments ${ }^{28}$. About a quarter of all Po synapses analyzed with FIB-SEM (9 out of 37) occur in nonvaricose "inter-bouton” axonal zones (S1-L5a: 5/20; S1-L1:1/4; MC-L4/3: 3/13).

Several such synapses are indicated by arrows in Fig. 4a-c. In VPM S1-L4 axons, synapses in non-varicose regions are much less frequent ${ }^{25,27 .}$

\section{Ultrastructural features of elements postsynaptic to the Po axons}

In all three cortical regions investigated, the majority of Po synapses were established on dendritic spines (83-96\%). Synapses onto dendritic shafts (which may partially correspond to non-spinous cortical interneurons ${ }^{29}$ ) were frequent $(17 \%)$ in S1-L5a, but less frequent by 4 -fold in S1-L1 or MC-L4/3 (4-6\%, respectively; Table 1; Fig. 2-4) with no contacts on neuronal somata.

Strikingly, the PSDs displayed a wide range in both shape and size (Fig. 4a'-c', Table 1). The mean surface area of the S1-L5a PSDs was comparable $\left(0.11 \mu \mathrm{m}^{2}\right)$ to that 
208

209

210

211

212

213

214

215

216

217

218

219

220

221

222

223

224

225

226

227

228

229

230

231

232

233

234

235

236

237

238

239

240

241

previously measured in VPM-L4 synapses (Table 1, Fig. 3g). Surprisingly, PSDs surface area of MC-L4/3 and S1-L1 Po synapses were $\sim 60 \%$ larger. In S1-L5a, most PSDs (65\%) had disc-like morphologies. In contrast, most PSDs in S1-L1 (57\%) or MC-L4/3 (59\%), displayed more complex horseshoe-shaped, perforated or fragmented morphologies (Fig. 4a'-c').

A total of 199 dendritic spines postsynaptic to Po boutons were analyzed (Table 1). Interestingly, some of them (3\%-18\%) were also targeted by an unlabeled symmetric (putatively inhibitory) synapse of unknown origin (Table 1). Only 30\% of the dendritic spines postsynaptic to Po boutons in S1 (L5a and L1) contained a spine apparatus (an endoplasmic reticulum derivate, Fig. 4a'-4b'), while the large majority (70\%) of postsynaptic spines in MC-L4/3 displayed this structural subelement responsible for spine motility but also stabilization of the pre-and postsynaptic apposition zone during synaptic transmission. Mitochondria were found inside 2 of the 68 dendritic spines postsynaptic to S1-L1 Po boutons, despite the exceedingly rare occurrence of spine mitochondria in rodent somatosensory cortex ${ }^{30}$.

\section{Insert Figure 5 about here}

\section{Dendritic spine invaginations into thalamocortical Po boutons}

Numerous dendritic spine heads postsynaptic to Po boutons formed a thick finger-like protrusion that invaginated into the presynaptic bouton (Fig. 5a-d). Such invaginations were observed in all three cortical domains investigated, but were more frequent in $\mathrm{MC}$ (Fig. 5e and Table 1). Invaginations were always adjacent to the spine PSD and present in spines with widely different PSDs sizes (Fig. 5e). Their volume was similar in the three cortical domains analyzed (range 0.6-0.75 $\mu \mathrm{m}^{2}$ ). The invaginated membranes were smooth, lacking evident membrane specializations (Fig. 5a 1 ). We had observed similar invaginations in mouse S1-L4 VPM synapses; remarkably their prevalence in Po MC synapses seems to be higher ${ }^{25}$ (13\% vs. 19\%; Fig. 5e-f).

\section{Quantitative comparisons of structural synaptic parameters}

To elucidate structural differences between Po axon synapses, parameters were quantitatively compared between the three cortical domains studied (Fig. 3d-h; Table 1). This analysis revealed striking differences. For example, Po boutons in MC-L4/3 were found to be significantly larger ( $\sim 60 \%$ in both volume and surface area) than Po 
242 boutons in S1-L5a (Fig. 3d). Most boutons in L1 were small, although the difference in

243

244

245

246

247

248

249

250

251

252

253

254

255

256

257

258

259

260

261

262

263

264

265

266

267

268

269

270

271

272

273

274

average size was less apparent due to the presence of occasional large boutons interspersed in the axonal branches (Supplementary Fig. SM4). Mitochondrial volume per Po bouton was $\sim 33 \%$ larger in the MC-L4/3 than in the S1-L5a boutons, consistent with their bigger size (Fig. 3e). Most importantly, boutons in MC-L4/3 and S1-L1 contained vesicle pools about twice as large as those in S1-L5a (Fig. 3g). In addition, both the head volume and the PSD surface area of the spines postsynaptic to Po boutons in MC-L4/3 and S1-L1 were significantly larger $(+50 \%)$ than those of spines postsynaptic to S1-L5a Po boutons (Fig. 3g-h).

Next, we compared the structure of synapses established by Po vs. VPM thalamic nuclei axons in $\mathrm{S} 1$ by putting side by side the 3D Po bouton measurements and those of VPM S1-L4 boutons ${ }^{25}$. This comparison revealed that the Po-L5a boutons are $-46 \%$ less in volume, and $-33 \%$ in mitochondrial volume and contained $-57 \%$ less synaptic vesicles than VPM-L4 boutons (Fig. 3d-f). In contrast, the Po MC-L4/3 boutons are statistically indistinguishable from VPM S1-L4 boutons with respect to these three parameters. Overall, this analysis demonstrates that a) axons from different thalamic nuclei can form structurally different presynaptic specializations in adjacent layers of the same cortical columns and b) axons from the same thalamic nucleus can form structurally different specializations in separate areas/layers.

In contrast, S1 spines postsynaptic to VPM (L4) and Po (L5a) boutons showed almost identical head volumes and PSD sizes. These two parameters were different, and overall much larger, for spines postsynaptic to S1-L1 ( $+83 \%$ and $+45 \%$, respectively) or MC-L4/3 (+50\% and 54\%) Po boutons (Fig. 3h), consistent with the notion that postsynaptic element differences reflect to a larger extent the local idiosyncrasies of distant neuropils.

Finally, to detect possible associations between pairs or groups of features in the structure of the Po boutons, we conducted a correlation $\left(\mathrm{R}^{2}\right)$ and cluster analyses of the structural parameters analyzed. Synapses in non-varicose axonal zones were not included. These analyses revealed that in all cortical areas, particularly in MC L3/4, the volume of Po synaptic boutons correlates with that of their resident mitochondria, but much less so with the size of their vesicle pool (Supplementary Fig. SM5 and SM6). In each neuropil, pre- and postsynaptic parameters clustered independently; for example, the PSDs surface area and number were only weakly or not correlated with bouton size, 
275 but PSD surface area is positively correlated to the volume of the spine head

276 (Supplementary Fig. SM5 and SM6).

277

278

Insert Figure 6 about here

279

280

Varicosities in the MC branches of Po axons are consistently larger than those in

281 branches of the same axons in $S 1$

282

For the 3D electron microscopic study, relatively large populations of Po cell axons

were anterogradely labeled with BDA. Since studies in rat indicate that MC and S1 may

284

be targeted by axonal branches of the same Po neuron ${ }^{4,24}$, it remained unclear whether

285

structural differences of Po synapses in MC and S1 reflect a) two different Po cell

286

populations, each projecting either to $\mathrm{MC}$ or to $\mathrm{S} 1$, or b) area-specific synaptic

287

structures in the collaterals of the same Po neuron. To address this question, isolated

288

individual Po neurons were transfection-labeled with a Sindbis-pal-eGFP RNA

289

construct using in vivo electroporation. From a larger collection of fully-reconstructed

290

Po neurons projecting to a variety of cortical territories ( $n=12$, data not shown), three

291

cells were found to specifically innervate vibrissal motor cortex; remarkably, all three

292

cells had, in addition, a collateral axon branch arborizing in the vibrissal region of the

293 primary somatosensory cortex.

The arborization of two of these cell axons in S1 and MC are illustrated in Fig. 6; the third is shown in Supplementary Fig. SM7. The laminar distribution of these individual axons in the cortex was similar to that produced by bulk-labeling with BDA iontophoresis. Under light-microscopic analysis, MC-L4/3 boutons in the three neurons were consistently larger $\left(1.13 \pm 0.43 \mu \mathrm{m}^{2}, 1.13 \pm 0.74 \mu \mathrm{m}^{2}\right.$ and $1.42 \pm 0.49 \mu \mathrm{m}^{2}$, respectively) than those formed by the branches of the same axons in S1-L5a (0.74 \pm $0.30 \mu \mathrm{m}^{2}, 0.83 \pm 0.39 \mu \mathrm{m}^{2}$ and $\left.0.96 \pm 0.35 \mu \mathrm{m}^{2}\right)$ or S1-L1 $\left(0.73 \pm 0.37 \mu \mathrm{m}^{2}\right.$ and $0.91 \pm$ size distributions revealed that the differences were highly significant (Fig. 6j-m).

304 Hence, our analysis shows that the separate axonal branches of individual Po neurons

305 form structurally different boutons in MC and S1. 


\section{Discussion}

308

309 Here, we demonstrate that individual Po neuron simultaneously innervate MC and S1

310 through branched axons that have varicosities (putative synaptic contacts) of markedly

311 different size in each area. These structural differences were found to be even more

312 pronounced using high-end 3D-electron microscopy: the Po boutons in MC-L4/3 differ

313 significantly from Po boutons in S1 (L1 and L5a) in volume, surface area, and mean

314 number of synaptic contacts. Moreover, MC-L4/3 and S1-L5a Po synapses are

315 significantly different in vesicle pool size, in PSD surface area and shape, and in their

316 proportion of synapses established on spines. Interestingly, about a quarter of the Po

317 synapses occur in non-varicose axonal segments. Comparison with our previous data on

318 VPM S1-L4 synapses ${ }^{25}$ reveals both a sharp contrast between Po and VPM synapses in

319 S1, which correlate with recently discovered differences in the functional properties of

320 these synapses, as well as intriguing similarities between Po synapses in MC and VPM

321 synapses in S1 (Table 1, Fig. 7).

322

Insert Figure 7 about here

324

Individual Po axons form structurally different synapses in MC and S1

326 Thalamic inputs reach the cortex via a diverse array of overlapping axonal pathways.

327 New techniques that make it possible to label the complete axonal arborization of

328 individual neurons are revealing that axons from most thalamic nuclei branch to target

329 several cortical areas (reviewed by ${ }^{31}$ ). In rats, individual Po cell axons can innervate

330 both the somatosensory and motor cortices, and often other regions ${ }^{4,24}$. In a recent

331 study of anterogradely bulk-labeled mouse Po axons, we showed that axonal

332 varicosities in MC-L4/3 are, as a population, significantly larger than varicosities in S1-

333 L5a ${ }^{3}$. Here, using single-cell transfection-labeling of single Po neurons and quantitative

334 axonal and bouton 3D-volume reconstructions, we demonstrate that these large MC-

335 L4/3 and small S1-5a varicosities occur on separate branches of individual Po axons,

336 and dovetail much deeper differences in synaptic 3D ultrastructure.

337 The most striking differences between Po MC-L4/3 axon synapses and S1-L5a is the

338 difference in their mitochondrial volume, PSD area and number of synaptic vesicles.

339 Large bouton and mitochondria volume and large vesicle pools are all linked to the

340 elevated energy supply and/or calcium homeostasis required to maintain high release 
341 rates ${ }^{28,32,33 ; 34}$. Mitochondria boost local ATP generation and control $\mathrm{Ca}^{+2}$ levels which

342 allow enhanced mobilization and recycling of synaptic vesicles for exocytosis,

343 neurotransmitter release and for the generation of synaptic membrane potentials,

344 specially under repetitive high-frequency firing ${ }^{33}$. Likewise, the presence of large

345 vesicle pools and extensive and complex PSDs is associated with higher

346 neurotransmitter release probabilities and synaptic efficacy $17 ; 18 ; 19 ; 35$ and to the number

347 and distribution of postsynaptic receptors $20,22,23$. The striking differences in these

348 parameters between Po MC-L3/4 and S1-L5a boutons thus imply that the first may

349 readily keep a high release probability at high-frequency firing rates, which is consistent

350 with the observed capacity of MC Po synapses to transmit signals with higher efficacy

351 and temporal acuity than Po S1 synapses ${ }^{3,16}$. Besides the structural differences, specific

352 ionotropic and metabotropic receptor distributions may contribute to the temporal

353 profile divergence of $\mathrm{MC}$ and $\mathrm{S} 1$ neuron responses to the repetitive activation of Po 354 synapses ${ }^{3}$.

355 Thalamocortical shaft synapses in rodent neocortex correspond in a large proportion

356 to contacts on cortical inhibitory neurons ${ }^{36 ; 29}$, but see ${ }^{37}$. Thus, our data indicate that,

357 mouse Po axons contact S1-L5a cortical interneurons in a proportion comparable to

358 other thalamocortical systems and species, including VPM-L4 axons (Table 1, Fig. 7).

359 However, we found three times less dendritic shaft synapses in MC-L4/3, suggesting

360 that Po axons establish relatively fewer contacts on $\mathrm{MC}$ interneurons, a finding in

361 register with the reported scarcity of VGluT2-possitive boutons contacting smooth

362 dendritic shafts in mouse motor cortex ${ }^{29}$. Thalamocortical axon contacts on cortical

363 interneurons produce a powerful di-synaptic feedforward inhibition, in parallel with the

364 excitation, on cortical neurons ${ }^{38 ; 39 ; 40}$; a scarcity of Po shaft synapses in MC seem thus

365 to be consistent with our recent observation that $\mathrm{MC}$ unit responses are facilitated by

366 rapid repetitive Po axons activation, while the $\mathrm{S} 1$ responses become depressed ${ }^{3}$.

368 Po and VPM synapses in S1 are markedly different in structure

369 Comparison of Po axon S1 synapses with VPM axon S1 synapses (Fig. 7; see also ${ }^{25}$ )

370 reveals a remarkable contrast: both types of thalamocortical axon contact postsynaptic

371 shafts or spines in the same proportion, and their PSD sizes are similar. However, their

372 presynaptic ultrastructure is strikingly different. Virtually all (95\%) VPM synaptic

373 boutons were large, containing one or several mitochondria, large vesicle pools, and

374 most of them (53\%) contained more than one (up to four) active zones. Only $5 \%$ of the 
375 VPM synapses lacked a mitochondrion (interbouton synapses), and no VPM axonal 376 segment containing a mitochondrion inside lacked an active zone ${ }^{25,27}$ (Table 1; Fig. 7).

377 In other words, in VPM axonal arbors, all mitochondria were found near a synapse. In 378 contrast, Po boutons in S1-L5a were nearly 50\% smaller, contained 30\% smaller 379 mitochondrial volumes and 50\% smaller vesicle pools. Only 9\% were multisynaptic, 380 and these had, at most, two active zones. Moreover, about $25 \%$ of Po synapses (defined 381 by a PSD and a vesicle pool) were found in "non-varicose" axonal domains.

382 Remarkably, 7\% of the varicosities contained a mitochondrion but lacked an active zone. In Po axons, therefore, mitochondria are far less concentrated around synaptic sites, a pattern reminiscent of that observed in the Shaffer cortico-cortical collateral system of the hippocampus, where over $50 \%$ of synaptic sites lack a mitochondrion and $8 \%$ of axonal varicosities containing a mitochondrion had no synaptic contact ${ }^{28}$.

Po S1 and VPM L4 synapses evoke each markedly different temporal response profiles in cortical cells as a result of the selective involvement of different types of glutamate receptors: the VPM synapses involve only ionotropic receptors and their EPSCs depress markedly by repetitive stimulation ${ }^{9}$, while Po synapses involve both ionotropic and metabotropic receptors and display EPSCs-facilitation ${ }^{3,15}$. Interestingly, the different presynaptic mitochondrial content in VPM vs. Po synapses might also contribute to their different effects on S1 neurons. By boosting local $\mathrm{Ca}^{2+}$ and ATP levels, mitochondria actively promote the docking and/or fusion of synaptic vesicles ${ }^{33}$, ${ }^{41}$, as well as the transport of vesicles from the resting to the recycling and readily releasable pool ${ }^{42}$. As a result, mitochondria-rich synapses are capable of maintaining a high release probability over a wide range of firing rates ${ }^{34}$. Our observations of larger mitochondrial concentration near the VPM S1 synapses seems thus consistent with electrophysiological evidence that VPM synapses produce relatively large $(\sim 4.8 \mathrm{mV})$ excitatory postsynaptic potentials ${ }^{9}$ or currents ${ }^{10}$ with low failure rates ${ }^{11,12}$, while Po synapses in S1-L5a produce smaller postsynaptic currents ${ }^{10,15,16}$, that have slower rise and decay times ${ }^{14}$.

In addition, studies over the past decade have revealed that mitochondrial distribution along axonal trees is optimized to match the local metabolic demands of their synapses. Importantly, a significant fraction of axonal mitochondria may remain mobile in adult axon arbors (reviewed by ${ }^{33}$ ). Low ATP and high calcium levels 407 promote the docking and/or fusion of mobile mitochondria near highly active synapses ${ }^{41}$. From this perspective, the presence in S1 Po axons of many synapses without 
409

410

411

412

413

414

415

416

417

418

419

420

421

422

423

424

425

426

427

428

429

430

431

432

433

434

435

436

437

438

439

440

441

442

mitochondria and of mitochondria distant from synapses suggests that synaptic efficacy might be readily enhanced by the mobilization of mitochondria to particular synapses. In contrast, the pattern observed in in VPM S1 axons (high mitochondria concentration at synapses and absence of non-synaptic mitochondria) may not allow significant changes in efficacy though mitochondrial re-distribution. This scenario is consistent with the recently discovered capacity of Po S1-L5a synapses for delayed yet stable potentiation as a result of conditional adult learning, a capacity that is lacking in VPM S1-L4 synapses ${ }^{13}$.

Finally, the comparison of the MC Po axon presynaptic structure of with that of VPM S1 synapses reveals intriguing similarities (Fig. 7; Table 1), despite the fact that these two synapse populations arise from different thalamic nuclei and are located in widely separated cortical domains. Such overall resemblance is consistent with electrophysiological observations that both Po MC and VPM S1 synapses elicit similarly large EPSCs ${ }^{9,16}$, exhibit paired-pulse depression and involve only ionotropic receptors ${ }^{3,16}$. Remarkably, however, the size of the Po MC-L3/4 synapse PSDs is $60 \%$ larger than that of the VPM L4 synapses, suggesting that the former may have even greater high synaptic strength and release probability. Moreover, as pointed out earlier, these synapses may have their effects on cortical cells less temporally curtailed by feedforward inhibition ${ }^{3}$.

\section{Large non-synaptic spine intrusions are frequent in thalamocortical boutons}

We show that many Po and VPM boutons have an elongated, thick protrusion of the postsynaptic spine head invaginated into them. Two previous $2 \mathrm{D}$ electron microscopic studies of lateral geniculate nucleus axon synapses in the primary visual cortex of ferrets ${ }^{43}$ and tree shrews ${ }^{44}$ reported similar spine profiles, suggesting that spine intrusions may be common in mammalian thalamocortical synapses. While their precise functional significance remains to be determined; our data already provide some intriguing clues. For example, invaginations are always found adjacent to the spine active zone, yet seem not to be directly related to its size (Fig. 5e-f). They are substantially more prevalent in the Po MC-L3/4 and the VPM S1-L4 synapses than in the S1 Po synapses (L5a and L1), suggesting some relationship with the absence of metabotropic glutamate receptors. Moreover, the large (up to 20 times the size of the active zone) and narrow invaginated intermembrane space is bound to create non-linear diffusion conditions, free of glial scavenging, for neurotransmitters, trophic factors or 
443 other secreted molecules. It is even possible that the extensive patch of parallel and

444 closely apposed membranes might allow local electric field (ephaptic) conduction ${ }^{45}$

445 between the cortical spine and its thalamocortical bouton.

446 In conclusion, we have demonstrated here that differences in the composition of

447 synaptic structure underlie and explain the divergent responsivity and plasticity of MC

448 vs. S1 neurons to Po input ${ }^{3,16}$, as well as that of S1 neurons to VPM vs. Po inputs ${ }^{10,13}$.

449 Moreover, the evidence that these structural and functional differences actually occur

450 between synapses in separate branches of the same individual Po cell axons indicates

451 that current global models of thalamo-cortical wiring and interaction ${ }^{46,47}$ may require

452 revision.

453 


\section{Acknowledgements}

The authors gratefully acknowledge, Ms. Begoña Rodriguez and Ms. Marta Callejo for excellent technical help.

\section{Author Contributions}

JRM, FC, JHRL and CP designed the experiments, JRM, CP and MRT prepared the materials, JHRL, JDF, AM, AS and LAN provided or operated key equipment, JRM, CP, MRT, DCT, AS, RY and AR analyzed the data, JRM, FC, CP and RY prepared the figures and tables, FC, JRM, and JHRL wrote the paper with input from DCT, AR, AM and JDF.

\section{Competing Interests statement}

This work was supported by funding from the European Union's Horizon 2020 Research and Innovation Programme (Grant Agreement No.785907 HBP SGA2). Additional support was provided by Spain's Ministerio de Ciencia, Innovación y Universidades (BFU 2107-88549-P) to FC, and by grants of the Helmholtz Society (JHRL).

All the authors declare that they have no other financial interests that might be relevant to the submitted study.

The data that support the findings of this study are available from the corresponding author upon reasonable request. In addition, all serial electron microscope image stacks and the two short videos cited in Supplemantary Materials Figure SM3 will be made available before publication in the Human Brain project Graph Data Platform https://www.humanbrainproject.eu/explore-the-brain/search" under a CC-BY license. 


\section{References}

489

490 01.- Ahissar, E. \& Oram, T. Thalamic relay or cortico-thalamic processing? Old 491 question, new answers. Cer. Cortex 25, 845-848 (2015).

492 02.- Wimmer, V.C., Bruno, R.M., de Kock, C.P., Kuner, T., Sakmann, B. Dimensions 493 of a projection column and architecture of VPM and POm axons in rat vibrissal cortex.

494 Cer. Cortex 20, 2265-2276 (2010).

495 03.- Casas-Torremocha, D. et al. Posterior thalamic nucleus axon terminals have 496 different structure and functional impact in the motor and somatosensory vibrissal 497 cortices. Brain Struct. Funct. 224,1627-1645 (2019).

498 04.- Ohno, S. et al. A morphological analysis of thalamocortical axon fibers of rat 499 posterior thalamic nuclei: a single neuron tracing study with viral vectors. Cereb. 500 Cortex 22, 2840-2857 (2012).

501 05.- Hooks, B.M., Lin, J.Y., Guo, C., Svoboda, K. Dual-channel circuit mapping reveals 502 sensorimotor convergence in the primary motor cortex. J. Neurosci. 35, 4418-4426 503 (2015).

504 06.- Yu, C., Derdikman, D., Haidarliu, S., Ahissar, E. Parallel thalamic pathways for 505 whisking and touch signals in the rat. PLoS Biology 4, e124 (2006).

506 07.- Groh, A. et al.. Convergence of cortical and sensory driver inputs on single 507 thalamocortical cells. Cereb.Cortex 24, 3167-3179 (2014).

508 08.- Mo, C., Petrof, I., Viaene, A.N., Sherman, S.M. Synaptic properties of the 509 lemniscal and paralemniscal pathways to the mouse somatosensory thalamus. Proc.

510 Natl. Acad. Sci. U.S.A. 114, E6212-E6221 (2017).

511 09. - Lee, C.C. \& Sherman, S.M. Synaptic properties of thalamic and intracortical 512 inputs to layer 4 of the first- and higher-order cortical areas in the auditory and 513 somatosensory systems. J. Neurophysiol. 100, 317-326 (2008).

514 10.- Petreanu, L., Mao, T., Sternson, S.M., Svoboda, K. The subcellular organization of 515 neocortical excitatory connections. Nature 457, 1142-1145 (2009).

516 11.- Gil, Z., Connors, B.W., Amitai, Y. Efficacy of thalamocortical and intracortical 517 synaptic connections: quanta, innervation, and reliability. Neuron 23, 385-397 (1999). 
518 12.- Bruno, R.M. \& Sakmann, B. Cortex is driven by weak but synchronously active 519 thalamocortical synapses. Science 312, 1622-1627 (2006).

520 13.- Audette, N.J., Bernhard, S.M., Ray, A., Stewart, L.T., Barth, A.L. Rapid plasticity 521 of higher-order thalamocortical inputs during sensory learning. Neuron 103, 277-291 522 (2019).

523 14.- Bureau, I., von Saint Paul, F., Svoboda, K. Interdigitated paralemniscal and 524 lemniscal pathways in the mouse barrel cortex. PLoS Biology 4, e382 (2006).

525 15.- Viaene, A.N., Petrof ,I., Sherman, S.M. Properties of the thalamic projection from 526 the posterior medial nucleus to primary and secondary somatosensory cortices in the 527 mouse. Proc. Natl. Acad. Sci. U.S.A. 108, 18156-18161 (2011).

528 16.- Mo, C. \& Sherman, S.M. A sensorimotor pathway via higher-order thalamus. $J$. 529 Neurosci. 39, 692-704 (2019).

530 17.- Geinisman, Y. Perforated axospinous synapses with multiple, completely 531 partitioned transmission zones: probable structural intermediates in synaptic plasticity. 532 Hippocampus 3, 417-433 (1993).

533 18.- Matz, J., Gilyan, A., Kolar, A., McCarvill, T., Krueger, S.R. Rapid structural 534 alterations of the active zone lead to sustained changes in neurotransmitter release.

535 Proc. Natl. Acad. Sci. U.S.A. 107, 8836-8841 (2010).

536 19. - Holderith, N. et al. Release probability of hippocampal glutamatergic terminals 537 scales with the size of the active zone. Nat. Neurosci. 15, 988-997 (2012).

538 20. - Nusser, Z. et al. Cell type and pathway dependence of synaptic AMPA receptor 539 number and variability in the hippocampus. Neuron 21, 545-559 (1998).

540 21. - Kharazia, V.N. \& Weinberg, R.J. Immunogold localization of AMPA and NMDA 541 receptors in somatic sensory cortex of albino rat. J. Comp. Neurol. 412, 292-302 (1999).

542 22. - Ganeshina, O., Berry, R.W., Petralia, R.S., Nicholson, D.A., Geinisman, Y.

543 Differences in the expression of AMPA and NMDA receptors between axospinous

544 perforated and nonperforated synapses are related to the configuration and size of 545 postsynaptic densities. J. Comp. Neurol. 468, 86-95 (2004). 
546 23.- Tarusawa, E. et al. Input-specific intrasynaptic arrangements of ionotropic

547 glutamate receptors and their impact on postsynaptic responses. J. Neurosci. 29, 12896-

$54812908(2009)$.

549 24.- Noseda, R., Jakubowski, M., Kainz, V., Borsook, D., Burstein, R. Cortical

550 projections of functionally identified thalamic trigeminovascular neurons: implications

551 for migraine headache and its associated symptoms. J. Neurosci. 31, 14204-14217

552 (2011).

553 26.- Peters, A. \& Palay, S.L. The morphology of synapses. J. Neurocytol. 25, 687-700

554 (1996).

555 27.- White, E.L., Weinfeld, E., Lev, D.L. Quantitative analysis of synaptic distribution 556 along thalamocortical axons in adult mouse barrels. J.Comp.Neurol. 479, 56-69 (2004).

557 25.- Rodriguez-Moreno, J. et al. Quantitative 3D ultrastructure of thalamocortical 558 synapses from the "lemniscal" ventral posteromedial nucleus in mouse barrel cortex.

559 Cereb. Cortex 28, 3159-3175 (2018).

560 28.- Shepherd, G.M. \& Harris, K.M. Three-dimensional structure and composition of

561 CA3 $\rightarrow$ CA1 axons in rat hippocampal slices: implications for presynaptic connectivity

562 and compartmentalization. J. Neurosci. 18, 8300-8310 (1998).

563 29.- Bopp, R., Holler-Rickauer, S., Martin, K.A., Schuhknecht, G.F. An ultrastructural 564 study of the thalamic input to layer 4 of primary motor and primary somatosensory 565 cortex in the mouse. J. Neurosci 37, 2435-2448 (2017).

566 30.- Santuy, A. et al. Quantitative study on the distribution of mitochondria in the 567 neuropil of the juvenile rat somatosensory cortex. Cereb. Cortex. 28, 3673-3684 568 (2018).

569 31.- Clasca, F., Porrero, C., Galazo, M., Rubio-Garrido, P., Evangelio, M. Anatomy and 570 development of multi-specific thalamocortical axons: implications for cortical dynamics 571 and evolution. In: Rockland KS (ed) Axons and brain architecture, 69-92 (Elsevier, 572 Amsterdam, 2016).

573 32.- Kharazia, V.N. \& Weinberg, R.J. Glutamate in thalamic fibers terminating in layer 574 IV of primary sensory cortex. J. Neurosci. 14, 6021-6032 (1994).

575 33. Sheng, Z.H. \& Cai, Q. Mitochondrial transport in neurons: impact on synaptic 576 homeostasis and neurodegeneration. Nat. Rev. Neurosci. 13, 77-93 (2012). 
577 34.- Cserép, C., Pósfai, B., Schwarcz, A.D., Dénes, Á. Mitochondrial ultrastructure is 578 coupled to synaptic performance at axonal release sites. eNeuro 29, 5 (2018).

579 35.- Valden, J.D., Banumurthy, G., Gusarevich, E.S., Overstreet-Waldiche, L.,

580 Waldiche, J.I. The readily-releasable pool dynamically regulates multivesicular release.

581 eLife 8, e47434 (2019).

582 36.- Staiger, J.F., Zilles, K., Freund, T.F. Distribution of GABAergic elements

583 postsynaptic to ventroposteromedial thalamic projections in layer IV of rat barrel

584 cortex. Eur. J. Neurosci. 8, 2273-2285 (1996).

585 37.- Silver, R.A., Lubke, J., Sakmann, B., Feldmeyer, D. High-probability uniquantal 586 transmission at excitatory synapses in barrel cortex. Science 302, 1981-1984 (2003).

587 38.- Agmon, A. \& Connors, B.W. Correlation between intrinsic firing patterns and 588 thalamocortical synaptic responses of neurons in mouse barrel cortex. J. Neurosci. 12, 589 319-329 (1992).

590 39.- Gabernet, L., Jadhav, S.P., Feldman, D.E., Carandini, M., Scanziani, M.

591 Somatosensory integration controlled by dynamic thalamocortical feed-forward

592 inhibition. Neuron 48, 315-327 (2005).

593 40.- Cruikshank, S.J., Lewis, T.J., Connors, B.W. Synaptic basis for intense 594 thalamocortical activation of feedforward inhibitory cells in neocortex. Nat.Neurosci. 595 10, 462-468 (2007).

596 41.- Saxton, W.M. \& Hollenbeck, P.J. The axonal transport of mitochondria. J. Cell Sci. 597 125, 2095-2104 (2012).

598 42.- Esposito, G., Ana Clara, F., Verstreken, P. Synaptic vesicle trafficking and 599 Parkinson's disease. Dev Neurobiol. 72, 134-44 (2012).

600 43.- Erisir, A. \& Dreusicke, M. Quantitative morphology and postsynaptic targets of 601 thalamocortical axons in critical period and adult ferret visual cortex. J. Comp. Neurol. $602485,11-31(2005)$.

603 44.- Familtsev, D. et al.. Ultrastructure of geniculocortical synaptic connections in the 604 tree shrew striate cortex. J. Comp. Neurol. 524, 1292-1306 (2016).

605 45.- Petralia, R.S., Wang, Y.X., Mattson, M.P., Yao, P.J. Invaginating structures in 606 mammalian synapses. Front. Synaptic Neurosci. 10, 4 (2018). 
607 46.- Jones, E.G. Viewpoint: the core and matrix of thalamic organization. Neuroscience 608 85, 331-345 (1998).

609 47.- Sherman, S.M. \& Guillery, R.W. Distinct functions for direct and transthalamic 610 corticocortical connections. J. Neurophysiol. 106, 1068-1077 (2011).

611 48.- Paxinos, G. \& Franklin K.. The mouse brain in stereotaxic coordinates. 4th ed 612 (Academic Press, Amsterdam-Boston, 2012). 


\section{Methods}

617

618

\section{Animals \& anesthetic procedures}

619 Experiments were performed on adult (60-105 days old, 25-32 g in body weight) male C57BL/6 mice bred in the Autónoma de Madrid University colony. All procedures involving live animals were conducted under protocols approved by the University ethics committee and the competent Spanish Government agency (PROEX175/16), in accordance with the European Community Council Directive 2010/63/UE. Animals were housed in pairs in cages containing some toys, and provided chow and water ad libitum under a 12 hours light/dark cycle. Efforts were made to minimize the number of animals required. Six male mice (60-65 days in age) were used for the experiments aimed at BDA population-labeling of Po axons for light- and electron microscopy. Thirty-two further mice were electroporated with Sindbis Pal-eGFP RNA to reveal the full extent of individual Po cell axons.

For both types of experiment, mice were first anesthetized with an initial intraperitoneal injection of ketamine $(0.075 \mathrm{mg} / \mathrm{g}$ body weight $)$ and xylazine $(0.02 \mathrm{mg} / \mathrm{g}$ body weight), and then maintained with oxygenated isoflurane $(0.5-2 \%)$ throughout the surgical procedure. Animals recovered promptly after the interruption of the isofluorane flow, at the end of the surgical procedure. Ibuprofen $(120 \mathrm{mg} / \mathrm{l})$ was added to the drinking water to ensure analgesia during the survival period. At the time of sacrifice, animals were overdosed with sodium pentobarbital ( $0.09 \mathrm{mg} / \mathrm{g}$ body weight, i.p.).

\section{BDA iontophoresis for selective population-labeling of Po axons}

Animals were placed in a stereotaxic frame (Kopf Instruments, Tujunga, CA, USA). BDA10K (2.5\% in saline; Molecular Probes-Invitrogen, Carlsbad, CA, USA) was iontophoretically microinjected (positive tip, 0.7-0.8 $\mu \mathrm{A} ; 1 \mathrm{sec}$ on/off cycle, 30-40 min

642 duration) with a Midgard Precision Current Source (Stoelting, Wood Dale, IL, USA)

643 under stereotaxic guidance $(1.8 \mathrm{~mm}$ posterior, $1.3 \mathrm{~mm}$ lateral, and $2.7 \mathrm{~mm}$ ventral to

644 Bregma $^{48}$ ), using borosilicate micropipettes (WPI, Sarasota, FL, USA; outer tip 645 diameter: $7-10 \mu \mathrm{m})$. The micropipette was finally removed, and the muscle and skin 646 were sutured and disinfected with iodinated povidone. 
649 Following a 5-days survival, injected mice were perfused with phosphate-buffered

650 saline (0.1 M PBS) followed by a fixative of $4 \%$ paraformaldehyde and $0.1 \%$

651 glutaraldehyde in 0.1 M PB for $30 \mathrm{~min}$. After the perfusion, brains were removed from

652 the skull, and post-fixed by immersion for 1 hour at $4{ }^{\circ} \mathrm{C}$ in the same fixative. Two series

653 of parallel coronal sections (50 $\mu$ m-thicknesses) were cut with a Leica VT 1200S

654 vibratome (Leica Microsystems, Nussloch, Germany). Sections were then cryo-

655 protected by incubation in a sucrose solution (30\% in $0.1 \mathrm{M} \mathrm{PB})$ overnight, and were

656 then rapidly freeze-thawed in liquid nitrogen $(1 \mathrm{~min})$ and stored in PB until further use.

657 In the first series of sections, peroxidase activity was blocked by incubation in PB-

658 buffered $\mathrm{H}_{2} \mathrm{O}_{2}$ for $10 \mathrm{~min}$, and sections were then incubated in an avidin-biotin-

659 peroxidase kit (1:100; Vectastain Elite ${ }^{\mathrm{TM}}$, Vector Laboratories, Burlingame, CA, USA)

660 diluted in PB. After washing in PB, labeling was visualized using the glucose oxidase-

661 3-3'diaminobenzidine (DAB) nickel sulfate-enhanced method ${ }^{49}$. Sections were

662 mounted onto gelatin-coated glass slides, counterstained with thionin, dehydrated in

663 graded ethanol, defatted in xylene, and coverslipped with DePeX (Serva, Germany).

664 These sections were used for light microscopic analysis (Nikon Eclipse 600, 4-40X

665 objectives) of the cytoarchitectonic localization of the injection site in the thalamus and

666 the anterogradely labeled axons in the neocortex. Out of the twelve BDA injected

667 hemispheres, four showed BDA injections restricted to Po (Fig. 1A) and robust axonal

668 labeling in S1 and MC (Fig. 1, Supplementary Fig. SM1). The two cortical areas and

669 respective target layers were identified based on the thionin counterstaining. The

670 vibrissal region of the MC as defined in this study is located along the border between

671 cytoarchitectonic areas AgM/M2 and AgL/M1 of the frontal cortex ${ }^{3,5}$ (Bregma AP:

$672+0.5-+2.2 \mathrm{~mm}$, ML: 1-1.5 mm). The vibrissal domain of S1 is readily identifiable by

673 its barrels in L4.

674 The second series of sections followed a protocol identical to that described above,

675 except for the omission of the oxygen-peroxide blocking step and the absence of the

676 nickel sulfate-enhancement in the glucose-oxidase-DAB reaction. In the four injection

677 experiments that produced optimal labeling of Po axons (see above), free-floating

678 sections from this series containing the regions with the cortical labeling underwent

679 further processing for EM. In these sections, the glucose oxidase-DAB reaction was

680 followed by incubation in 1\% osmium tetroxide (Electron Microscopy Science,

681 Hartfield, PA, USA) diluted in PB for $45 \mathrm{~min}$ at room temperature. Following thorough

682 washing in $\mathrm{PB}$, sections were first rinsed in 50\% ethanol, incubated for $40 \mathrm{~min}$ in $1 \%$ 
683

684

685

686

687

688

689

690

691

692

693

694

695

696

697

698

699

700

701

702

703

704

705

706

707

708

709

710

711

712

713

714

715

716

uranyl-acetate diluted in 70\% ethanol in the dark, and dehydrated in an ascending series of ethanol to absolute ethanol. The dehydrated sections were transferred to acetonitrile (Scharlab, Barcelona, Spain), and then transferred to an epoxy resin (Durcupan ${ }^{\mathrm{TM}}$, Electron Microscopy Science, Hartfield PA, USA) overnight. Finally, sections were flat-embedded in Durcupan ${ }^{\mathrm{TM}}$ and polymerized at $60^{\circ} \mathrm{C}$ for $48 \mathrm{hrs}$.

After light microscopic inspection, samples containing dense labeling in the S1 and $\mathrm{MC}$ neocortex were cut out and glued onto pre-polymerized resin blocks for serial section and subsequent ssTEM imaging (Supplementary Fig. SM2).

\section{Tissue processing for ssTEM imaging of BDA-labeled Po boutons}

Embedded tissue blocks that contained L5a and L1 in S1 barrel cortex or L4/3 in MC were cut with a Leica Ultracut UCT ultramicrotome (Leica Microsystems, Nussloch, Germany) into serial $60 \mathrm{~nm}$ ultrathin sections (around 90 sections/series). They were collected on pioloform-coated single-slot copper grids (Electron Microscopy Science). Thereafter, they were treated with lead citrate ( $3 \mathrm{~min}$ ), and examined with a Libra 120 transmission electron microscope (EM, Fa. Carl Zeiss, Oberkochen, Germany) equipped with a Proscan $2 \mathrm{~K}$ bottom-mounted digital camera (Fa. Albert Tröndle, Moorenweis, Germany) and the SIS Multi Image Acquisition software package (Olympus, Hamburg, Germany). At the EM level, BDA-labeled axons were easily identifiable by the opaque DAB reaction product. Serial digital images were taken at a magnification of $8000 \mathrm{x}$ and stored in a database until further use.

\section{FIB-SEM 3D tissue preparation and imaging of BDA-labeled Po boutons}

In addition, some Durcupan-embedded tissue blocks from the same experiments were used to obtained 3D tissue samples using combined focused gallium ion beam milling and scanning electron microscopy (FIB-SEM). Here, a Crossbeam Neon40 EsB electron microscope equipped with a gallium FIB and a high-resolution scanning emission SEM column (Carl Zeiss) was used. In order to accurately select the regions of interest, a secondary electron microscopic image was acquired from the block surface that was overlaid and collated with previously obtained light microscopic images ${ }^{50}$. Once the appropriate location was chosen, a gallium ion beam was used to mill the sample to allow visualization of brain tissue under the block face on a nanometer scale. The recently milled surface was then imaged using a back-scattered electron detector (1.8 $\mathrm{kV}$ acceleration potential). The milling and imaging processes were sequentially 
717 repeated in an automated way, providing a stack of serial digital images that represented

718 a 3D sample of the tissue ${ }^{51}$. Image resolution in the xy-plane was $5 \mathrm{~nm}$ per pixel.

719 Resolution in the z-axis (section thickness) was $20 \mathrm{~nm}$, so the voxel size of the resulting 720 image stack was $5 \times 5 \times 20 \mathrm{~nm}$.

721 With the above resolution parameters, images of $2048 \times 1536$ pixels (field of view of $72210.24 \times 7.68$ microns) were obtained. A total of twenty different stacks of images of the 723 neuropil in the three cortical regions of interest were obtained (seven stacks from S1-

724 L5a, six from S1-L1, and seven from MC-L3/4). The number of serial sections per stack ranged from 75 to 478 ; the total number of serial sections was 4,874 (mean: 243.7 sections per stack). Registration (alignment) of serial sections was performed with the freely available Fiji software ${ }^{52}$, using a rigid body model that allowed no deformation of individual images.

\section{D-volume reconstruction and analysis of sSTEM and FIB-SEM image stacks}

The 3D-volume reconstructions and measurements on ssTEM images were carried out with the OpenCAR software (Contour Alignment Reconstruction) ${ }^{53}$. Digital images were aligned creating an image stack where all structures of interest were defined by closed contour lines of different color.

The Z-stack image series acquired with FIB-SEM were 3D-segmented and measured with the Espina Interactive Neuron Analyzer software (v.2.1.10; freely available at http://cajalbbp.es/espina/) ${ }^{54}$. 3D-reconstructions generated from separate Z-stacks were digitally stitched using Unity 3D modeling software (Unity Technologies, San Francisco, CA, USA).

The geometry of Po synaptic boutons, the PSD, mitochondria and the total pool of synaptic vesicles within individual boutons, and the postsynaptic target structures contacted by the synaptic boutons were completely 3D-reconstructed. From the contours, 3D-volume reconstructions were performed, from which surface area and volume measurements were obtained. The number of synaptic vesicles was estimated by a Physical Dissector stereological method ${ }^{55}$ in Po presynaptic boutons with a light DAB reaction product.

For each structural parameter, a mean \pm standard deviation (SD), the median, maximum and minimum values and a coefficient of variation $(\mathrm{CV})$ were calculated. For subsequent data analysis for multiple comparisons, we used one-way analysis of variance (ANOVA) plus T3 Dunnet's as a post hoc test with SPSS software (version 24; 
751 IBM, Armonk, New York). The threshold level of significance was set at $P<0.05$,

752

753

754

755

756

757

758

759

760

761

762

763

764

765

766

767

768

769

770

771

772

773

774

775

776

777

778

779

780

781

782

783

784 indicating this as (*), $P<0.01$ as $(* *)$ and $P<0.001$ as $(* * *)$.

\section{Cluster analysis comparison of synaptic bouton ultrastructural parameters}

All synaptic boutons reconstructed were clustered according to six structural parameters investigated, namely: bouton volume, bouton surface area, number of PSDs/bouton, number of mitochondria/bouton, volume of mitochondria and PSD surface area. The cluster analysis was performed using MATLAB and Statistics Toolbox Release 2016b (The MathWorks, Inc., Natick, MA, USA). The aim was to identify the synaptic parameters that best characterized the synaptic boutons investigated. Thus, a principal component analysis (PCA) was performed to simplify the dataset and to convert a set of observations of possibly correlated variables into a set of values of linearly uncorrelated variables called principal components (PCs) ${ }^{56}$. Then, we performed a hierarchical cluster analysis (HCA) ${ }^{57}$ on the new simplified dataset composed of the PCs. This method is used for unsupervised machine learning when the original data were unlabeled (For details see ${ }^{58}$ ).

\section{Sindbis-pal-eGFP RNA electroporation for single-cell labeling}

To directly visualize the complete axonal tree of individual mouse Po cells in an unambiguous manner, isolated Po neurons were transfected by means of in vivo electroporation ${ }^{59}$ of a RNA construct engineered to drive the expression of an enhanced green fluorescent protein (eGFP) fused with a palmytoilation motif from the growthassociated protein 43 (GAP43) under the Sindbis viral subgenomic promoter (Sind-PaleGFP) ${ }^{60}$.

Micropipettes were pulled from Kwick-Fill borosilicate capillaries ( $1 \mathrm{~mm}$ outer diameter; WPI). Inner tip diameter was adjusted to $10-15 \mu \mathrm{m}$. To eliminate RNAse activity, micropipettes were then kept in a stove overnight at $240^{\circ} \mathrm{C}$ and, after cooling, were backfilled with a RNA stock solution $(1.8-2 \mu \mathrm{g} / \mu 1$ in $0.5 \mathrm{M} \mathrm{NaCl})$ and mounted on a holder (WPI) that has both a pressure port and electrode connection. All procedures were performed over clean, single-use surfaces, and metal instrument tips were briefly exposed to a flame.

The micropipette tip was stereotaxically positioned into Po. 50-100 nl of solution were injected using a precision electro-valve system (Picospritzer II, Parker Hannifin, Cleveland $\mathrm{OH})$. Two to four $200 \mathrm{~Hz}$ trains of $1 \mathrm{~ms}$ negative-square pulses at $50 \mathrm{~V}$ were 
785

786

787

788

789

790

791

792

793

794

795

796

797

798

799

800

801

802

803

804

805

806

807

808

809

810

811

812

813

814

815

816

817

then applied through the micropipette tip using a CS20 stimulator (Cibertec, Madrid, Spain). The micropipette tip was left in place for $5 \mathrm{~min}$ before removing it from the brain. Finally, the bone defect was closed, the scalp sutured, and animals were returned to their cages.

Following 58-60 h survival, animals were overdosed with pentobarbital $(80 \mathrm{mg} / \mathrm{kg}$ body weight, i.p), and perfused with saline ( $1 \mathrm{~min}$ ), followed by $4 \%$ paraformaldehyde in $0.1 \mathrm{M} \mathrm{PB}, \mathrm{pH} 7.4$, for $8 \mathrm{~min}$. Their brains were then removed, immersed overnight in the same fixative at $4{ }^{\circ} \mathrm{C}$ and cryoprotected by soaking in a sucrose solution $(30 \%$ in PB) for further $24 \mathrm{hrs}$. Serial $50-\mu \mathrm{m}$-thick coronal sections were cut on freezing microtome.

To allow intensive high-magnification microscopic analysis, labeling was made stable and opaque by using immunohistochemistry against eGFP and glucose oxidasenickel enhancement ${ }^{49}$. Free-floating sections were incubated in rabbit anti-GFP serum (1:500; EXBIO, Prague, Czech Republic), followed by incubation with a biotinylated goat anti-rabbit serum (1:100; Sigma-Aldrich, St. Louis, MO, USA) and an avidinbiotin-peroxidase kit (1:100; Vectastain Elite, Vector Laboratories). Sections were then serially mounted onto gelatin-coated glass slides, air-dried, lightly counterstained with thionin, dehydrated and coverslipped with DePeX (Serva).

Sections were examined under brightfield optics at 10-40X. Labeled axons of Po neurons were found to span $>30$ serial coronal sections. Complete 2D-reconstructions of each axon were drawn using a Nikon camera lucida, scanned, and redrawn using a vector graphics software (Canvas X, ACD Systems, Saanichton BC, Canada).

Under bright-field optics, axonal branches appeared as sharply labeled filaments with frequent varicose swellings. To estimate and compare the size of varicosities (putative synaptic boutons), the maximal projection area was measured from live images using a Nikon DMX1200 camera fitted to the microscope and the NIS-Elements imaging software tools (v3.2; Nikon). To this end, the major perimeter of each varicosity was focused at 1000X and delineated over the computer screen using the "polyline" and "polygon" software tools. For each cortical area, layer and axon, 50 randomly selected varicosities were measured. Varicosities with cross-sectional areas (maximal projection) near or below the microscope resolution limit $\left(<0.2 \mu \mathrm{m}^{2}\right)$ we not included. Statistical analysis was performed with the SPSS software (IBM). Comparison of mean sizes of varicosities between areas/layers was performed using Mann-Whitney U test. Two- 
818

819

820

821

822

823

824

825

826

827

828

829

830

831

832

833

834

835

836

837

838

839

840

841 56.- Abdi, H. \& Williams, L.J. Principal component analysis. Wiley Interdiscip. Rev.

842 Comput. Stat. 2, 433-459 (2010).

843 57.- Rokach, L. \& Maimon, O. Clustering Methods. In: Maimon O., Rokach L. (eds)

844 Data Mining and Knowledge Discovery Handbook (Springer, Boston, MA, 2005).

sample Kolmogorov-Smirnov test (K-S) was used to compare size distributions of varicosities between areas/layers.

\section{Methods References}

49.- Shu, S.Y., Ju, G., Fan, L.Z. The glucose oxidase-DAB-nickel method in peroxidase histochemistry of the nervous system. Neurosci. Lett. 85, 169-71 (1988).

50.- Bosch, C. et al. FIB/SEM technology and high-throughput 3D reconstruction of dendritic spines and synapses in GFP-labeled adult-generated neurons. Front.

Neuroanat. 9, 60 (2015).

51.- Merchán-Pérez, A., Rodríguez, J.R., Alonso-Nanclares, L., Schertel, A., DeFelipe, J. Counting synapses using FIB/SEM microscopy: a true revolution for ultrastructural volume reconstruction. Front. Neuroanat. 3, 18 (2009).

52.- Schindelin, J. et al. Fiji: an open-source platform for biological-image analysis. Nat. Methods 9, 676-682 (2012).

53.- Sätzler, K. et al. Three-dimensional reconstruction of a calyx of Held and its postsynaptic principal neuron in the medial nucleus of the trapezoid body. $J$ Neurosci. 22, 10567-10579 (2002).

54.- Morales, J. et al. Espina: a tool for the automated segmentation and counting of synapses in large stacks of electron microscopy images. Front. Neuroanat. 5, 18 (2011).

55.- Sterio, D.C. The unbiased estimation of number and sizes of arbitrary particles using the dissector. J. Microscopy 134, 127-136 (1984). 
845 58.- Yakoubi, R. et al. Quantitative three-dimensional reconstructions of excitatory

846 synaptic boutons in layer 5 of the adult human temporal lobe neocortex: a fine-scale

847 electron microscopic analysis. Cereb.Cortex 29, 2797-2814 (2019).

848 59.- Porrero, C. et al. A simple and efficient in vivo non-viral RNA transfection method

849 for labeling the whole axonal tree of individual adult long-range projection neurons.

850 Front. Neuroanat. 10, 27 (2016).

851 60.- Furuta, $T$. et al. In vivo transduction of central neurons using recombinant Sindbis

852 virus: Golgi-like labeling of dendrites and axons with membrane-targeted fluorescent

853 proteins. J. Histochem Cytochem. 49, 1497-1508 (2001). 

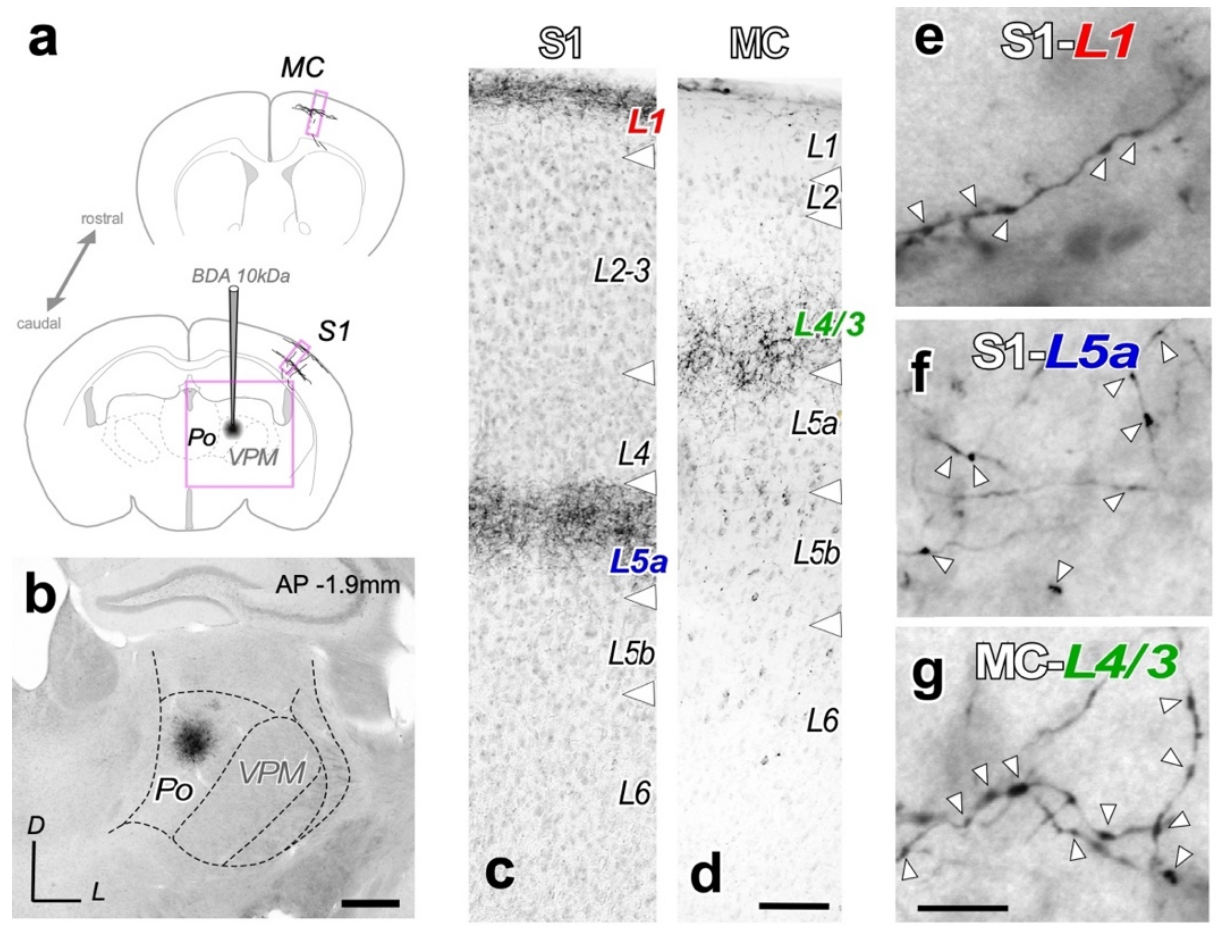

859

Fig. 1. Selective bulk-labeling of Po thalamocortical axons in the vibrissal motor

861 (MC) and vibrissal somatosensory (S1) cortices. (a) BDA iontophoresis procedure. Schematic coronal brain sections depicting the BDA deposit in Po, and the anterogradely labeled thalamocortical axonal in S1 and MC. (b) A typical iontophoretic BDA Po deposit. The region illustrated is the area framed in panel "a". Thionin counterstain. Scale bar represents $250 \mu \mathrm{m}$. (c-d) Coronal sections showing the layer selective arborization of BDA-labeled Po axons in specific layers (L), namely L1 and L5a in S1 ("c") and L4/3 in MC ("d"). Scale bar represents $100 \mu \mathrm{m}$. (e-g) Highmagnification images of BDA-labeled Po axons in S1-L1. (e), in S1-L5a (f), and in MC-L4/3 (g). Axonal varicosities are marked by arrowheads. Scale bar represents 10 $\mu \mathrm{m}$. 

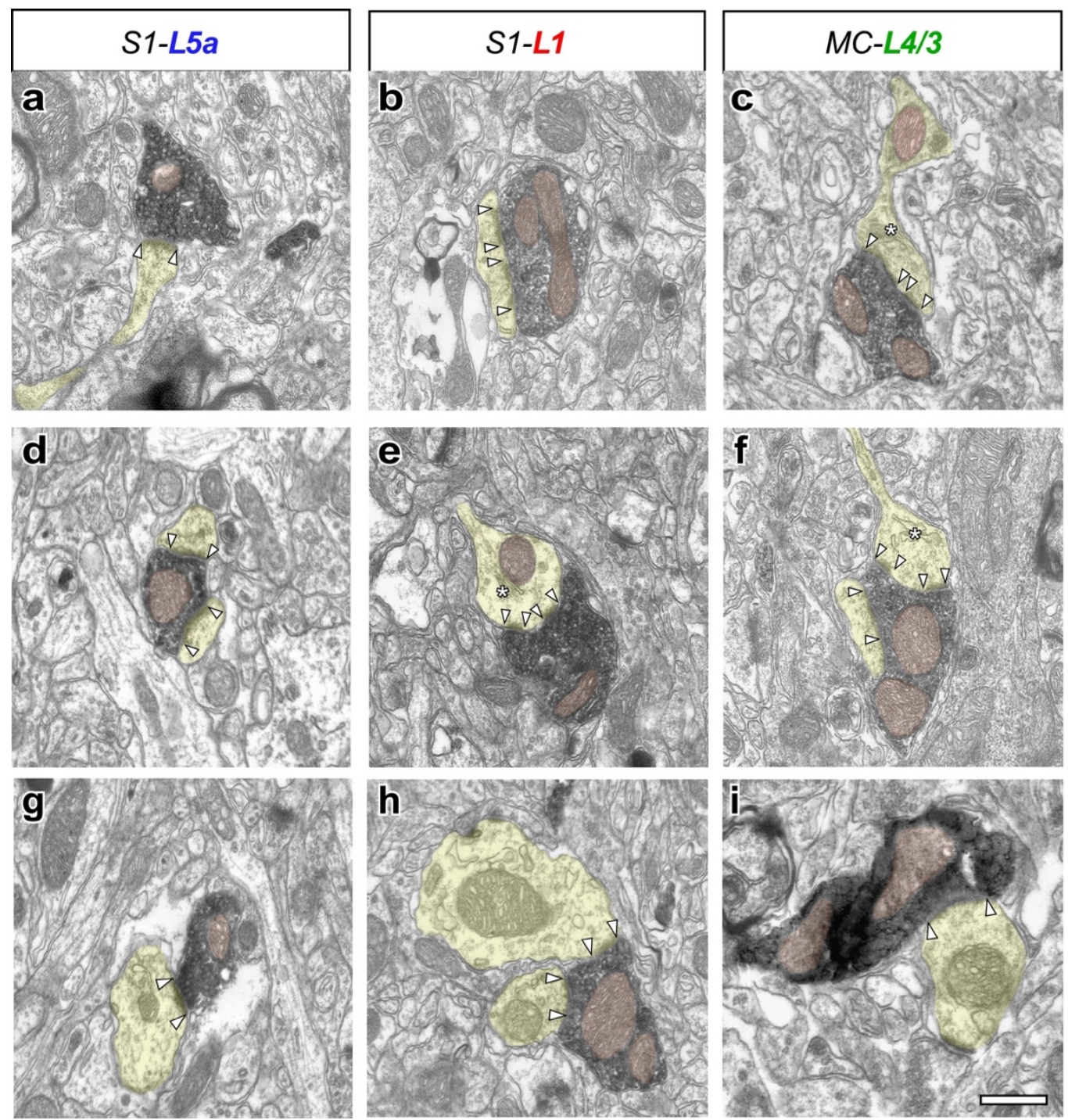

877

878 Fig. 2. Electron micrograph examples of BDA-labeled Po thalamocortical boutons establishing synaptic contacts with cortical dendritic spines or shafts. In all images,

880 dendritic shafts and spines are highlighted in transparent yellow and mitochondria are

881 shaded in transparent brown. PSD borders are marked by white arrowheads. Asterisks

882 indicate the spine apparatus. Inside some boutons, synaptic vesicles are clearly visible.

883 (a, d) Typical Po boutons synapsing on a dendritic spine head (a) or simultaneously onto two different spine heads (d) in S1-L5a. (b, e) Po boutons synapsing on dendritic spines in S1-L1. In e, note the presence of a mitochondrion and a spine apparatus (asterisk) within the postsynaptic spine head. (c, f) Synaptic Po boutons in MC-L3/4 synapsing onto one (c) or two dendritic spines simultaneously (f). (g-i) Examples of Po bouton contacts onto dendritic shafts, one in each of the three studied neuropils. Scale bar represents $0.5 \mu \mathrm{m}$. 

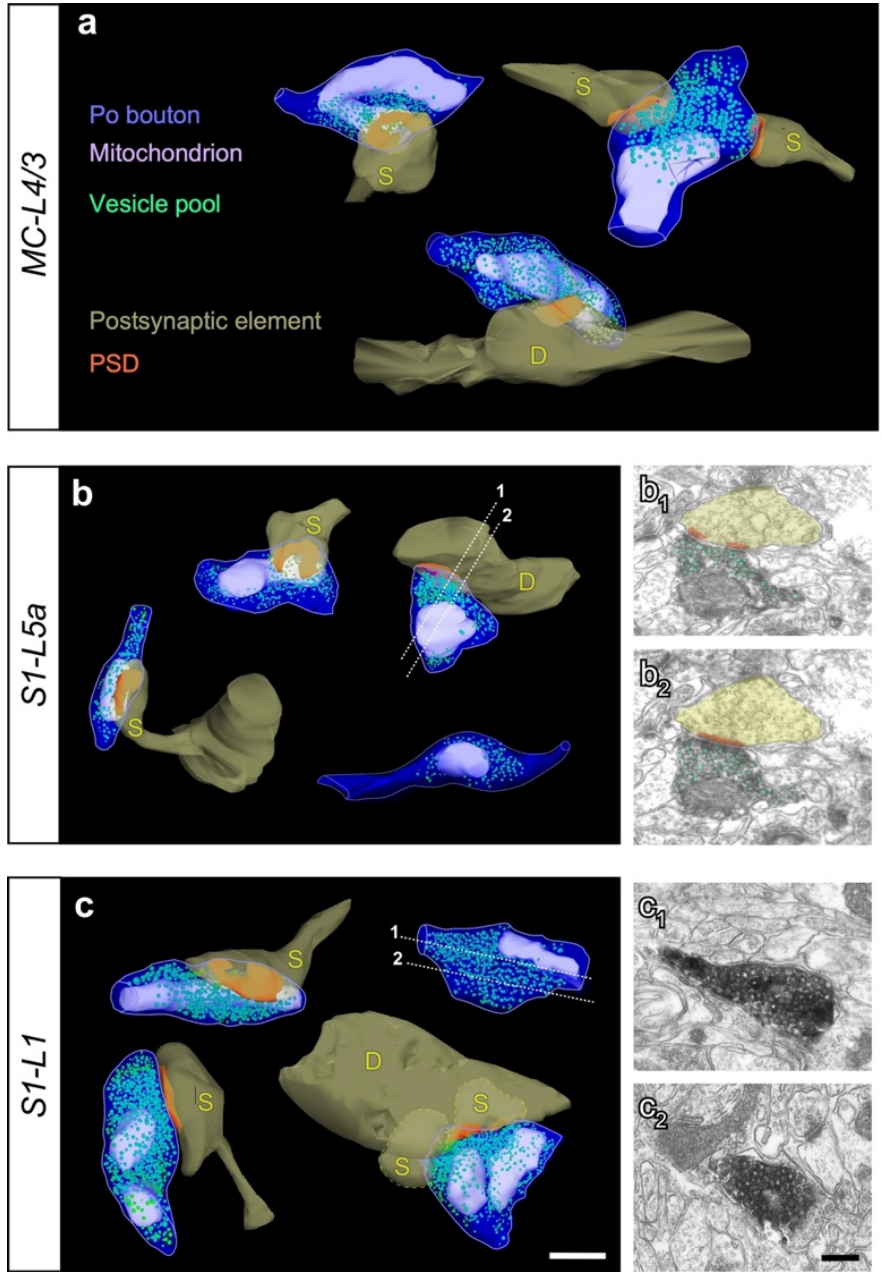

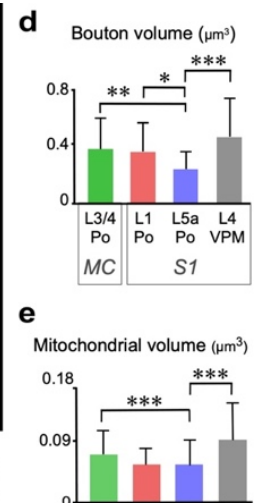

f

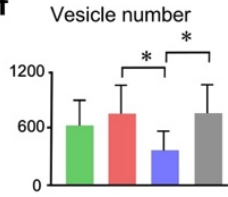

g PSD surface $\left(\mu \mathrm{m}^{2}\right)$

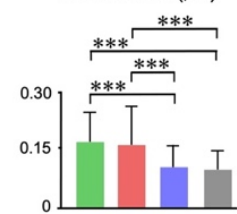

h

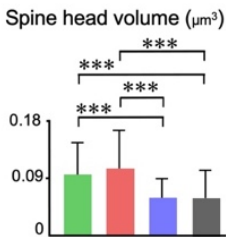

Fig. 3. ssTEM 3D-reconstructions of thalamocortical Po boutons, and comparison of their ultrastructural parameters in the various cortical domains studied. (a)

894 Boutons labeled in MC-L4/3. Top: Two boutons contacting spines ("S"). The bouton on the right is simultaneously establishing synaptic contacts with two different spines.

Bottom: a synaptic bouton terminating on a dendrite shaft ("D"). (b) Boutons labeled in S1-L5a. Left: two boutons contacting dendritic spines. Right (top): a bouton synapsing on a dendritic shaft (“D”). Two of the serial sections (indicated by dashed white lines) out of which this bouton was reconstructed are illustrated in panels $\mathbf{b}_{1}-\mathbf{b}_{2}$. Right

900 (bottom): an axon varicosity containing a mitochondrion and synaptic vesicles, but no 901 adjacent PSD or postsynaptic profile. (c) Boutons labeled in S1-L1. Left: two boutons establishing synaptic contacts with mushroom-like dendritic spines. Right (top): axonal varicosity containing a mitochondrion and synaptic vesicles, but no adjacent PSD. Two sections are shown in $\left(\mathbf{c}_{1}-\mathbf{c}_{2}\right)$. Right (bottom): a bouton simultaneously contacting a

905 dendritic shaft and two different spines. Scale bar represents $0.5 \mu \mathrm{m}$. (d-h) Bar 
907 vesicle number; (g) PSD surface area; and (h) spine head volume between Po synapses 908 in the three cortical neuropil areas investigated (colored bars). Measurements for VPM

909 synaptic boutons in S1-L4 (gray bar) are included (gray bar). Levels of significance are

910 indicated by asterisks: * $\mathrm{p} \leq 0.05 ; * * \mathrm{p} \leq 0.01 ; * * \mathrm{p} \leq 0.001$. 

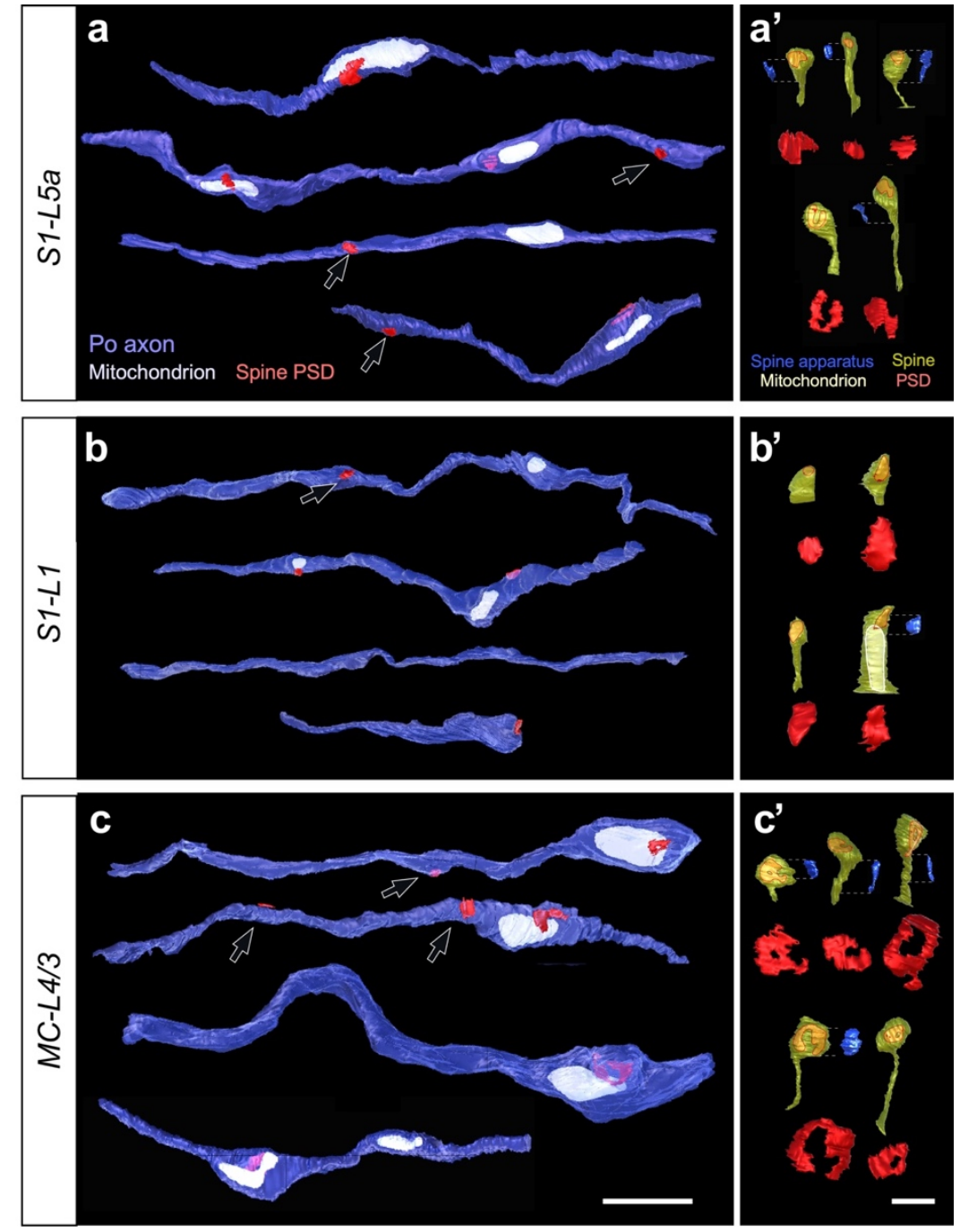

914 Fig. 4. Representative examples of thalamocortical Po axon segments, postsynaptic

915 dendritic spines and PSDs 3D-reconstructed from FIB-SEM image stacks. (a-c)

916 Individual axonal segments from (a) S1-L5a; (b) S1-L1, or (c) MC-L4/3. Black arrows

917 indicate PSDs located in non-varicose axon domains. Scale bar represents $2 \mu \mathrm{m}$. (a'-c')

918 Different morphologies of dendritic spines postsynaptic to Po boutons in (a') S1-L5a,

919 (b') S1-L1, and (c') MC-L4/3. Spine surface is partly transparent to allow the

920 visualization of the PSDs within the spine head. In the spines containing a spine

921 apparatus, this organelle (blue) is shown aside. Scale bar (for spines and spine

922 apparatuses) represent $0.5 \mu \mathrm{m}$. For clarity, the spine PSDs are also represented isolated

923 below, at double magnification. Note the differences in both shape (perforated vs. non-

924 perforated, closed ring- or horseshoe-like) and size of the PSDs. In (b') note the large

925 spine containing a mitochondrion. 

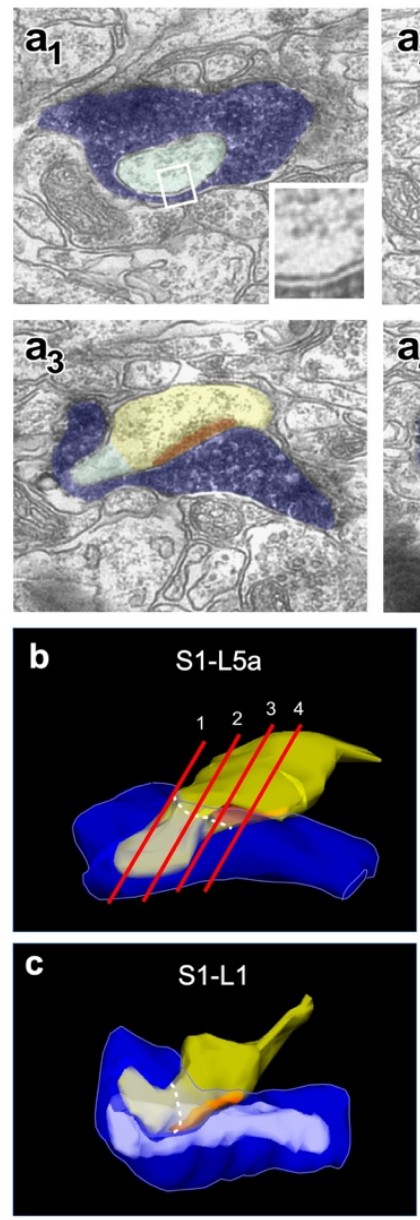
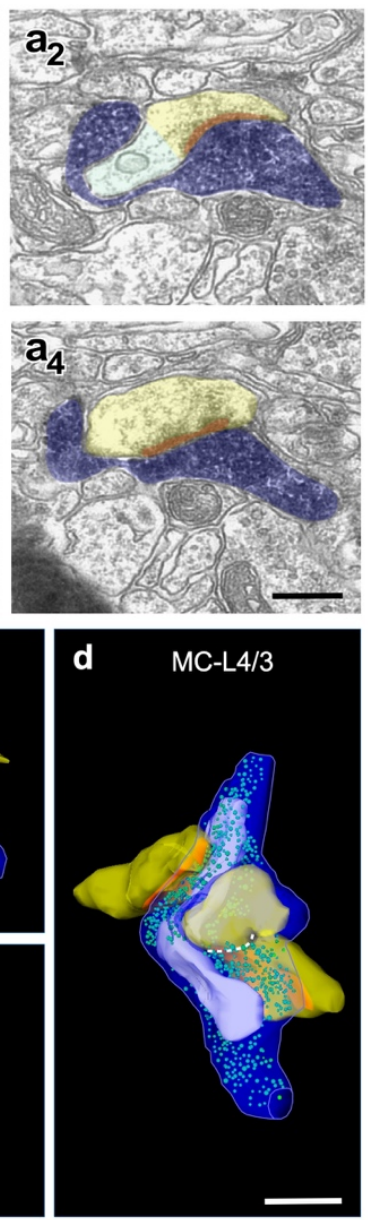

e

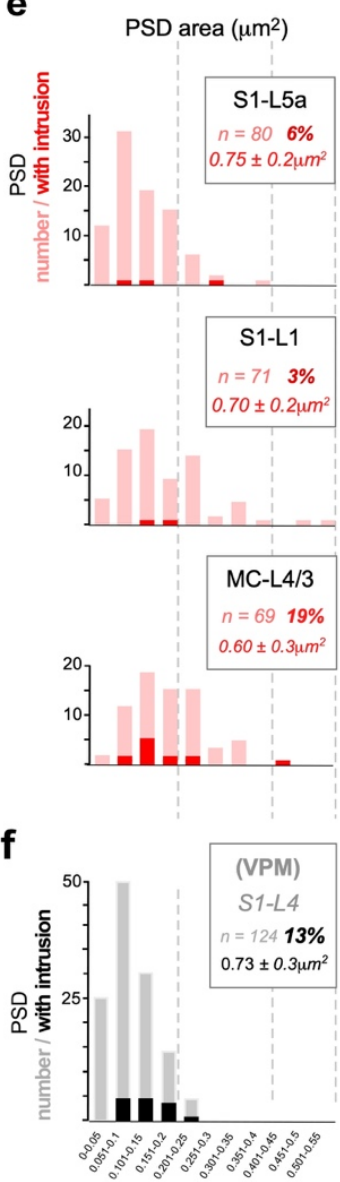

Fig. 5. Large cortical dendritic spine protrusions invaginate into the presynaptic

930 Po boutons. (a $\left.\mathbf{a}_{1}-\mathbf{a}_{4}\right)$ Consecutive electron micrographs showing a postsynaptic spine

931 head (transparent yellow) with a large protrusion (transparent green) invaginated into a

932 labeled Po bouton (transparent blue). Note that the PSD (red) is adjacent to the

933 protrusion, but not extending into it. Note also the close apposition of the pre- and

934 postsynaptic cell membranes in the intruded region (inset in $\mathbf{a}_{1}$ ). Scale bar represents 0.5

$935 \mu \mathrm{m}$. (b-d) 3D ssTEM reconstructed examples of synaptic boutons in which a cortical

936 spine protrusion is invaginated into a labeled presynaptic Po bouton. A dashed white

937 line highlights the approximate border between the spine protrusion and the remaining

938 spine head. The synaptic bouton in $(\mathbf{b})$ is a reconstruction made from the same series of

939 sections shown in (a1-a4) (red lines). Scale bar represent $0.5 \mu \mathrm{m}$. (e) Bar histograms

940 showing the distribution in PSD surface area in thalamocortical Po synaptic boutons in

941 each of the three cortical regions examined. Total numbers of PSD are represented in

942 pink. PSDs with a dendritic spine protrusion adjacent to them are highlighted in red. In

943 the box, the total number of PSD measured in each cortical region, the percentage of 
944 PSDs with adjacent protrusions, and the mean surface area \pm SD of the protrusion

945 membrane are indicated. (f) To allow a direct comparison, PSD surface areas and

946 protrusions measured in S1-L4 VPM synapses ${ }^{25}$ are displayed in gray/black.

947

948 

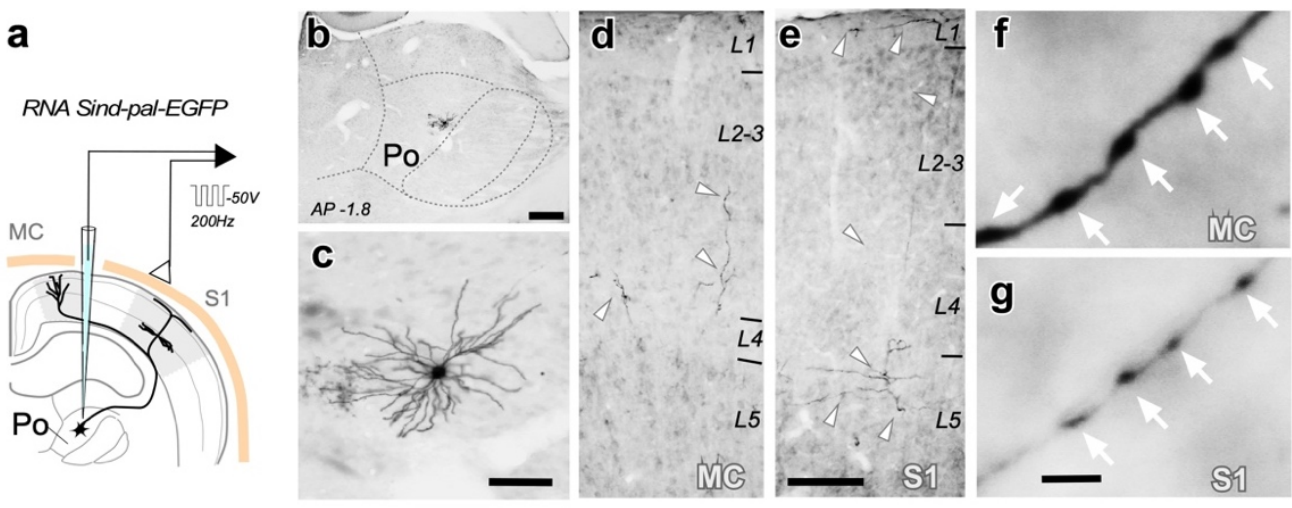

h

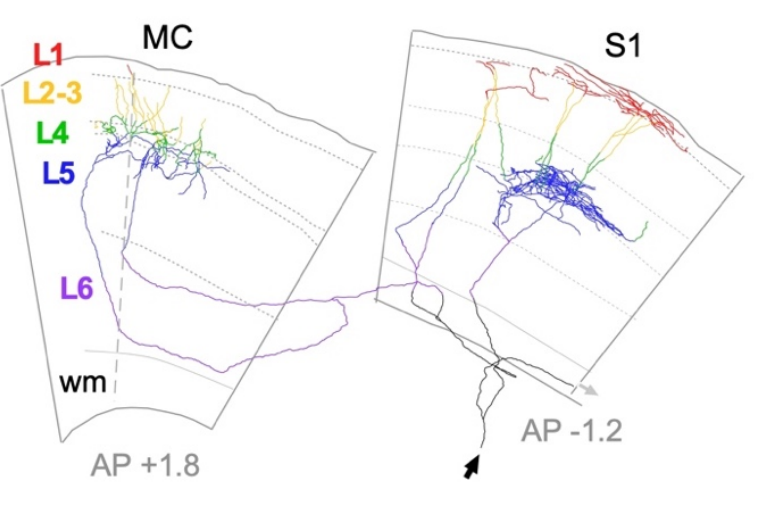

j
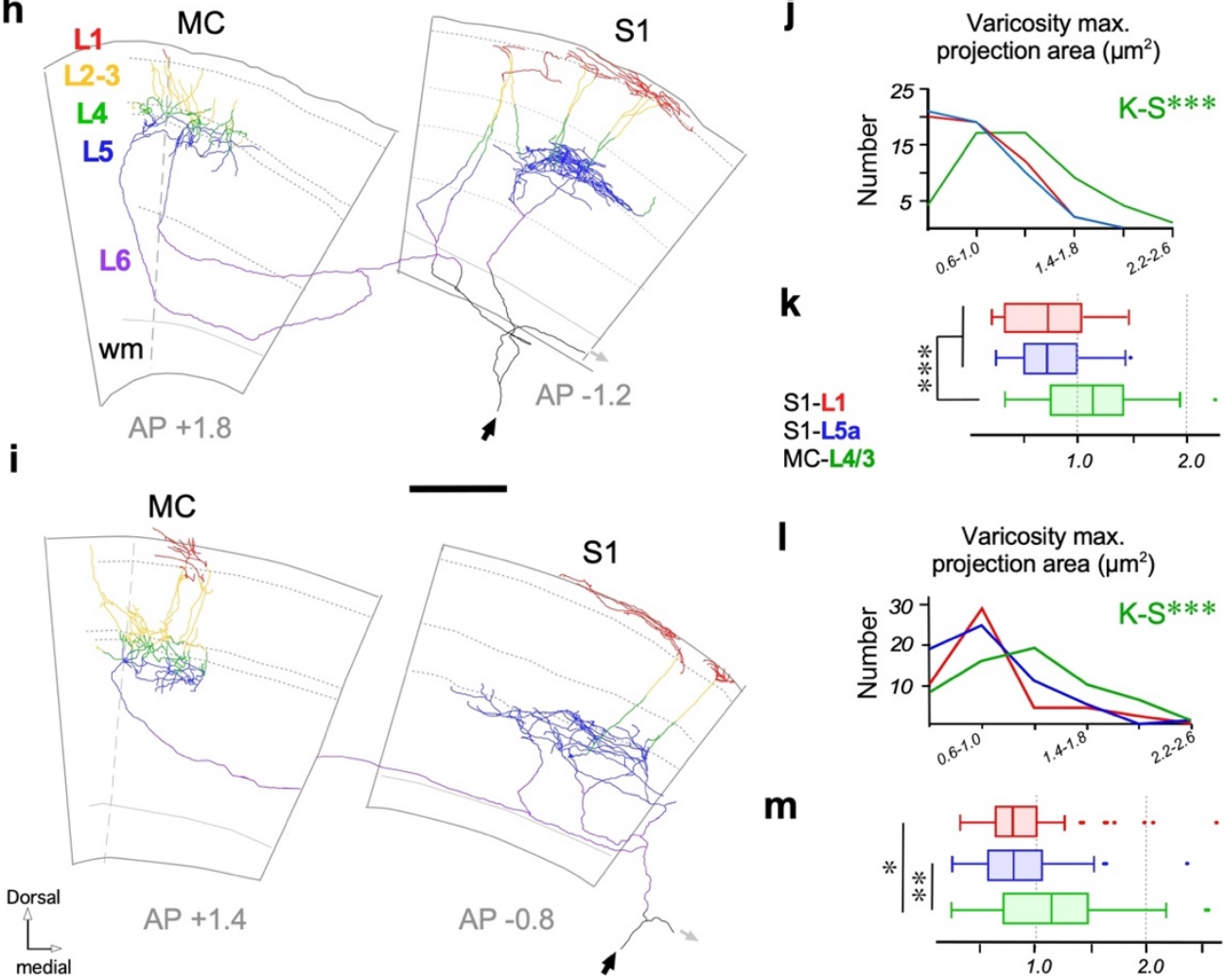

|

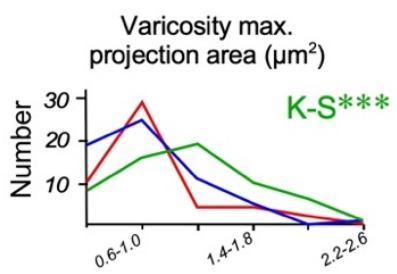

m

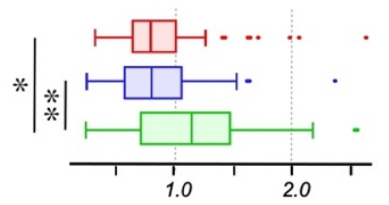

Fig. 6. Individual branched Po axons target simultaneously MC and S1 and have varicosities of significantly different size in each area. (a) Schematic diagram of the

953 Sindbis-pal-eGFP RNA electroporation procedure. (b) Coronal section through the

954 thalamus showing an isolated neuron transfected in Po. (c) Somatodendritic

955 morphology of the cell shown in "b". (d, e) Labeled thalamocortical axon fragments

956 (white arrowheads) as seen in columnar samples from MC (d) and S1 (e). (f, g) Po cell

957 axonal boutons in MC or S1 at high light microscopic magnification. Note the marked

958 differences in axonal diameter and varicosities sizes (white arrows). (h, i) Camera

959 lucida reconstructions of the axonal arborizations of two individually-labeled Po 
960 neurons targeting both MC and S1. Axonal domains located in the different cortical

961 layers or in the white matter (wm) are coded in different colors. Outlines of cortical

962 layers (grey lines) are shown as a background. A dashed line in MC indicates border

963 between areas Agl/M1 and Agm/M2 ${ }^{48}$. Distance to bregma is indicated. The point of

964 entry of the axon coming from the thalamus (black arrow) and a branch extending to

965 more lateral areas (not shown; gray arrow) are indicated. (j, l) Comparison of varicosity

966 size (maximal projection areas) distributions of the MC L4/3 vs. S1-L5a vs. S1-L1

967 branches of each axon. K-S = Kolmogorov-Smirnov. (k, m) Comparison of mean

968 maximal projection area. Mann-Whitney U Test. Scales bars: b $250 \mu \mathrm{m}$; c $50 \mu \mathrm{m}$; d, e

$969100 \mu \mathrm{m} ; \mathrm{f}, \mathrm{g} 5 \mu \mathrm{m}$; h, i $500 \mu \mathrm{m}$. 

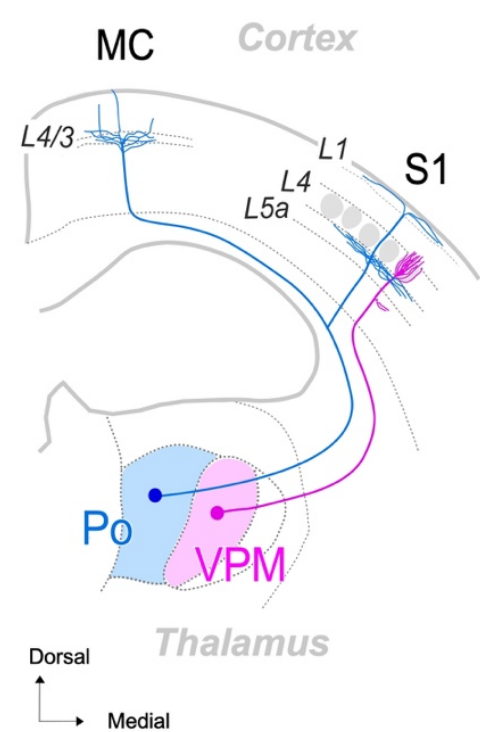

$\frac{M C}{L 4 / 3}$

Bouton volume $\left(\mu \mathrm{m}^{3}\right)$

Multisynaptic boutons (\%)

Non-synaptic boutons (\%)

Vesicle Pool size (mean)

Non-varicose synapses (\%)

Sinapses/ $\mu \mathrm{m}$ axon length

-.......-

Shaft synapses (\%)

PSD mean area $\left(\mu \mathrm{m}^{2}\right)$

Complex PSDs (\%)

Spine protrusions (\%)

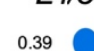

0.39

29

2

613

23

0.14

6

0.17

0.17

59

19

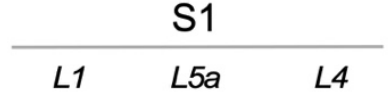

$0.37^{*} \quad 0.24$

$12 \bigcirc \quad 9 \quad 0.24 \bigcirc 5$

$7 \quad 7 \bigcirc 0$

$733 \bigcirc \quad 346 \bigcirc 740$

25

0.05

5

0.34

17

0.10

51

13

Po

972 Fig. 7. Graphic summary of relevant structural differences between Po axon

973 synapses in MC-L4/3 and S1 (L1, and L5a) and comparison with the S1-L4 VPM

974 axon synapses. The diagram on the left depicts, on a coronal mouse brain section, the

975 trajectory and cortical arborizations from a Po cell axon (blue) and a VPM cell axon

976 (magenta). The table on the right summarizes in simplified graphic format, to facilitate

977 comparisons, the most salient differences in synaptic structure observed in the present

978 study between Po boutons in MC-L4/3, S1-L1 and S1-L5a. For clarity, numbers are

979 rounded-up mean values (see Table 1 for details). The column on the right (magenta)

980 displays, with the same conventions, the values measured in S1-L4 VPM synapses.

981

982

983

984 
bioRxiv preprint doi: https://doi.org/10.1101/798926; this version posted October 9, 2019. The copyright holder for this preprint (which was not certified by peer review) is the author/funder. All rights reserved. No reuse allowed without permission.

985 Table 1: Ultrastructural 3D measurements of Po synapses in MC-L4/3, S1-L1 or S1986 L5a, and comparison with VPM synapses in S1-L4.

987

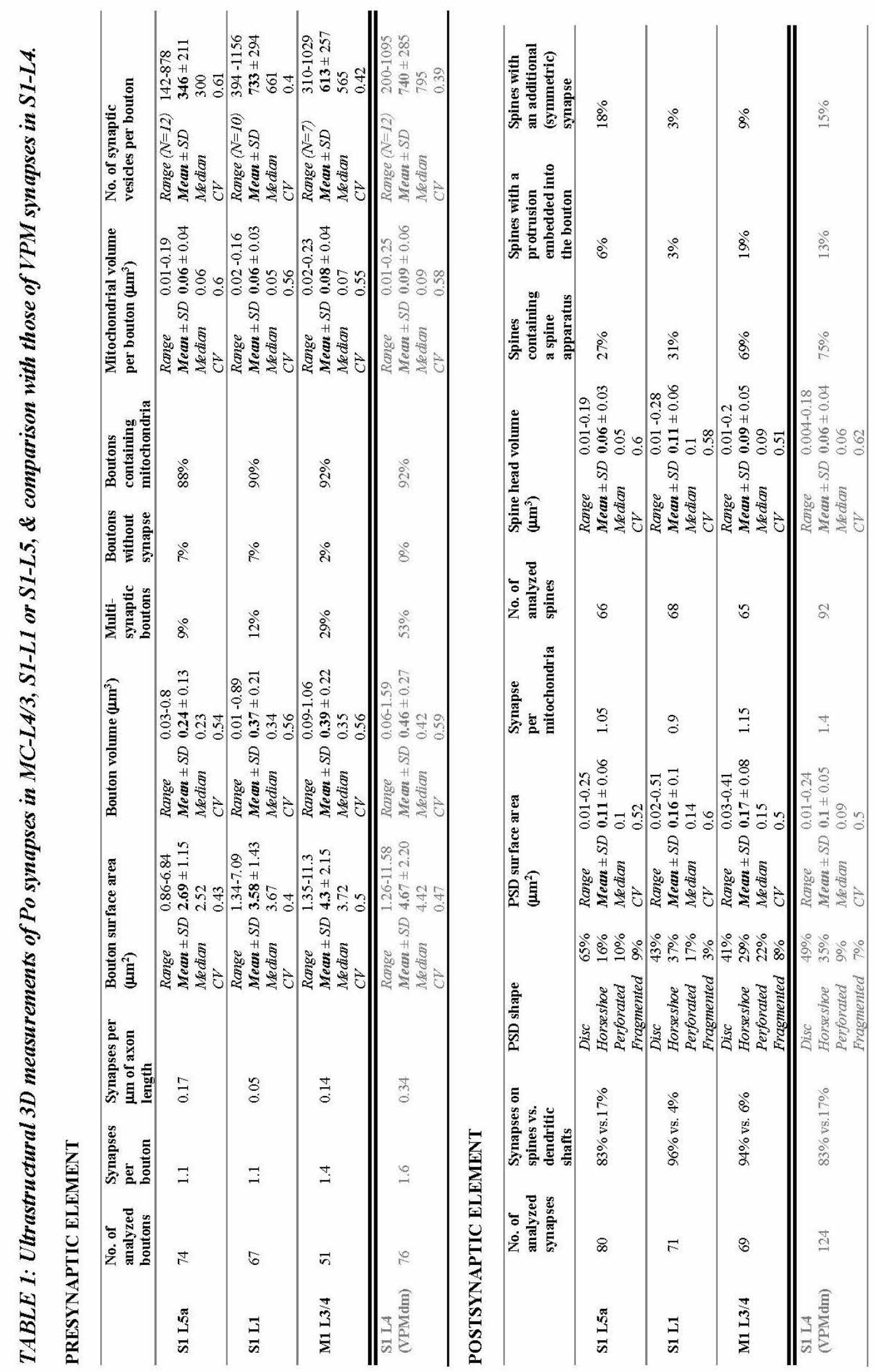


bioRxiv preprint doi: https://doi.org/10.1101/798926; this version posted October 9, 2019. The copyright holder for this preprint (which was not certified by peer review) is the author/funder. All rights reserved. No reuse allowed without permission.

989

990

991

992

993

994

995

996

Supplementary Information

997

998

999 

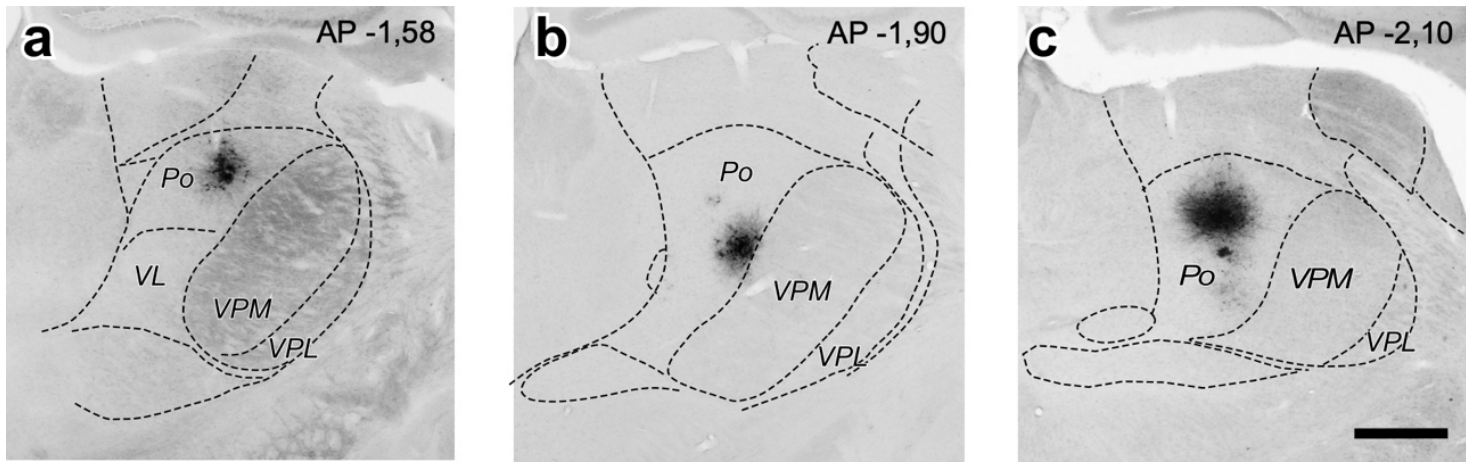

1006

1007 Supplementary Fig. SM1. Three further examples of BDA iontophoretic deposits used

1008 to selectively label Po thalamocortical axons in MC and S1. (a-c) Coronal thalamus

1009 sections show the center of the BDA deposit in Po. Light thionin counterstain. Distance

1010 (in $\mathrm{mm}$ ) caudal to bregma is indicated in the upper right corner. Scale bar represents

$1011250 \mu \mathrm{m}$.

1012 


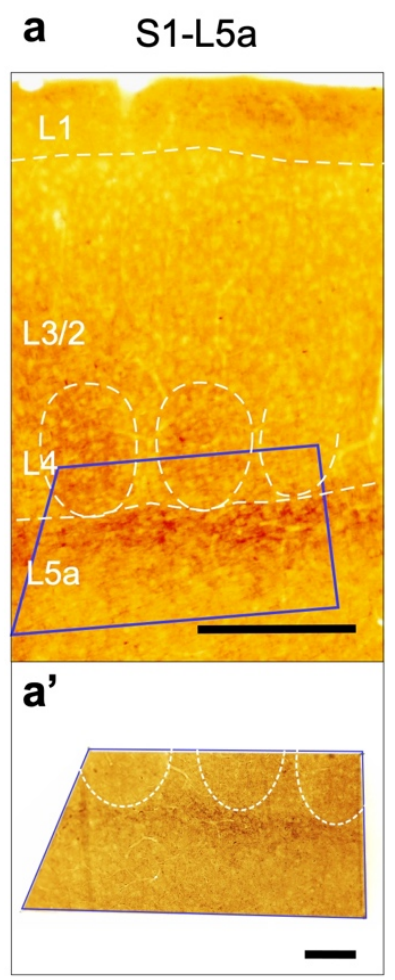

b S1-L1

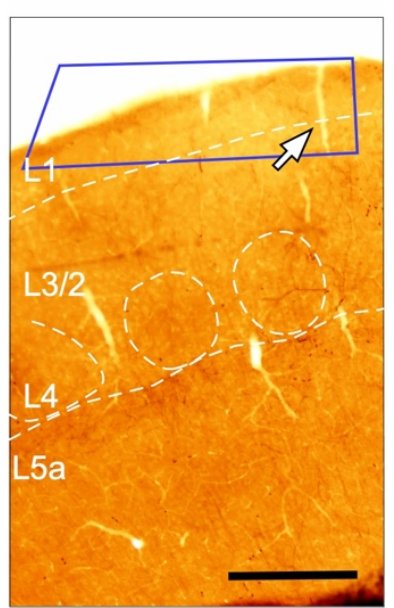

b'

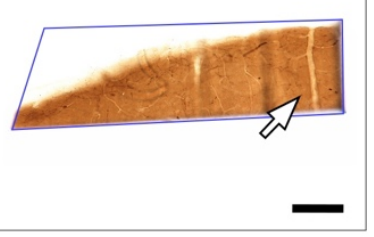

C $\quad$ MC-L4/3

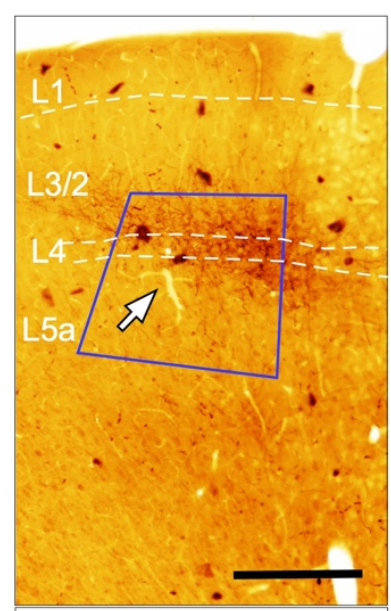

$\mathbf{C}^{\prime}$

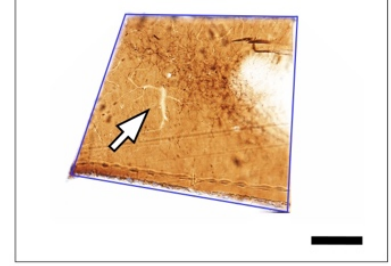

1013

1014 Supplementary Fig. SM2. Selective sampling of tissue areas containing BDA-labeled

1015 Po axon branches. (a-c) Resin-embedded coronal sections. The blue rectangle indicates

1016 the area that was cut out for subsequent EM serial sectioning. Scale bars represent 250

$1017 \mu \mathrm{m}\left(\mathbf{a}^{\prime}-\mathbf{c}^{\prime}\right)$. An arrow identifies the same vascular landmark in both images Scale bar

1018 represents $100 \mu \mathrm{m}$.

1019

1020 

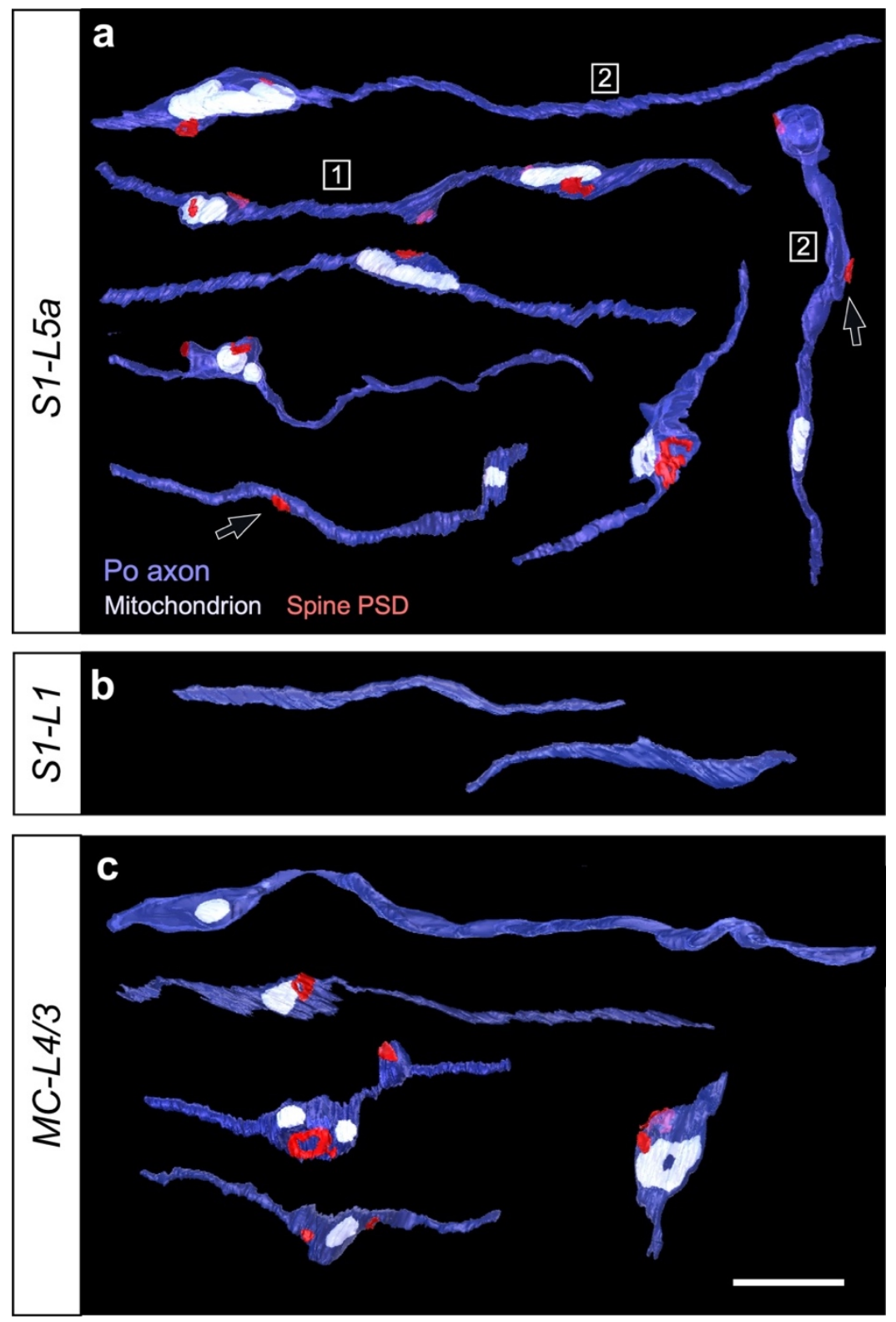

1023 Supplementary Fig. SM3. Additional thalamocortical Po axonal segments 3D-

1024 reconstructed from FIB-SEM image stacks. (a-c) Individual axonal segments from (a)

1025 S1-L5a, (b) S1-L1, or (c) MC-L4/3. All the PSDs in these particular segments were

1026 located on spines. Black arrows highlight PSDs located in non-varicose axonal

1027 domains. Axon segments labeled with numbers 1 and 2 are shown, within their

1028 correspondent 3D FIBSEM image stacks, in Supplementary videos SMV1 and SMV2.

1029 Scale bar represents $2 \mu \mathrm{m}$. 

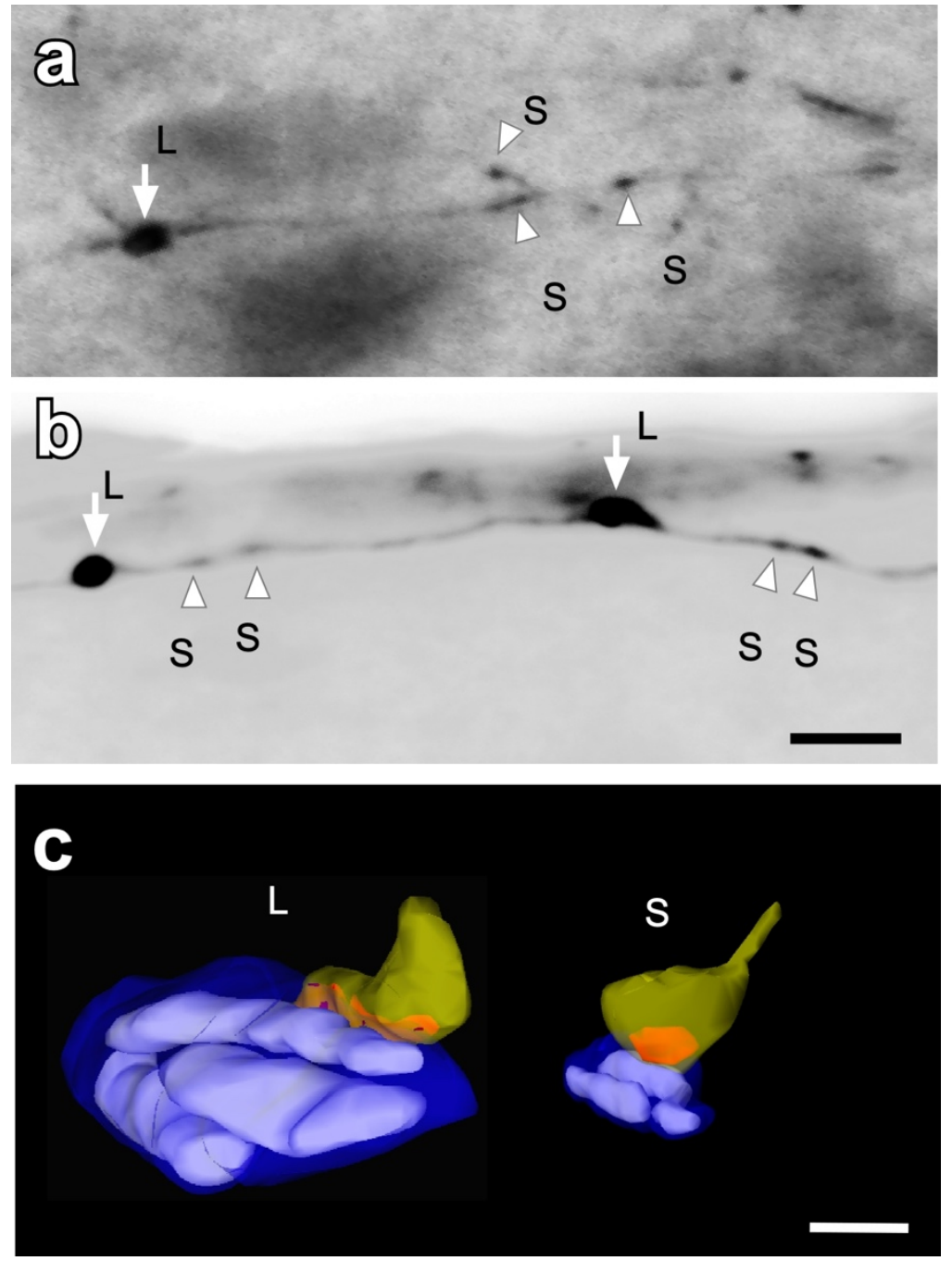

1033 Supplementary Fig. SM4. Po axon branches in S1bf L1 contain a majority of small synaptic

1034 boutons with occasional large boutons interspersed. (a-b) Light microscopy high-magnification 1035 images of a labeled Po axon containing both large bouton and small boutons. The branch in (a) 1036 was labeled by a BDA injection in Po. The branch in (b) was labeled by the transfection of an 1037 isolated Po neuron with Sindbis-pal-eGFP RNA. Scale bar represents $0.5 \mu \mathrm{m}$. (c)

1038 Representative examples of ssTEM 3D reconstructions of a larger (L) and smaller (S) Po axon 1039 boutons in S1-L1. Scale bar represents $2 \mu \mathrm{m}$. 
a

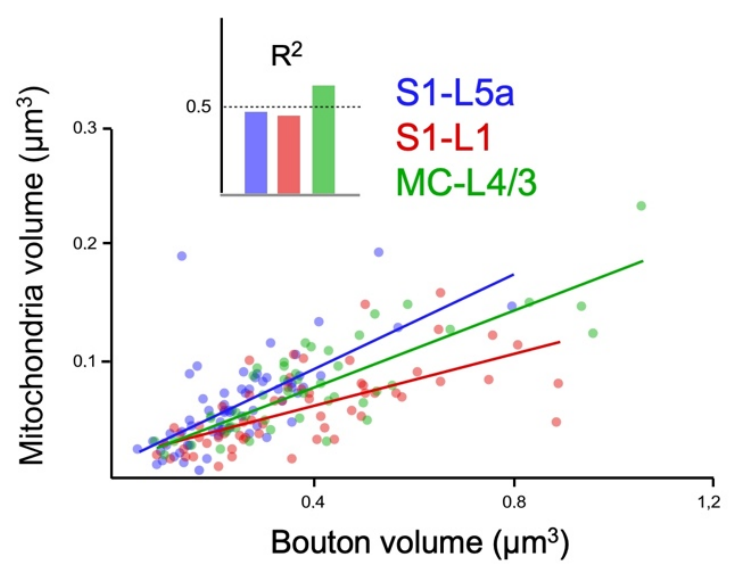

C

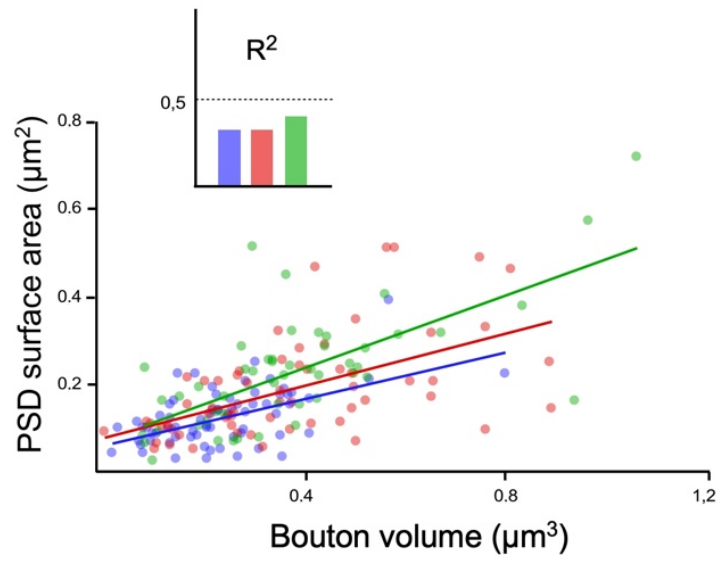

b

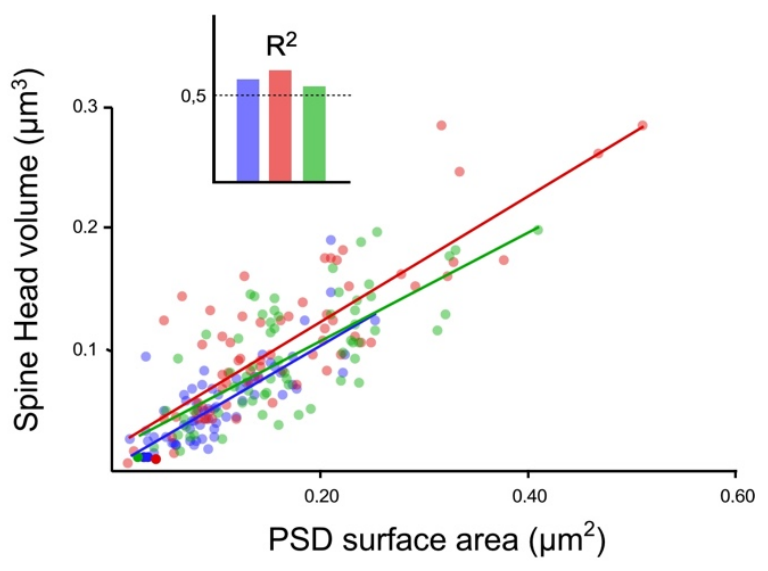

d

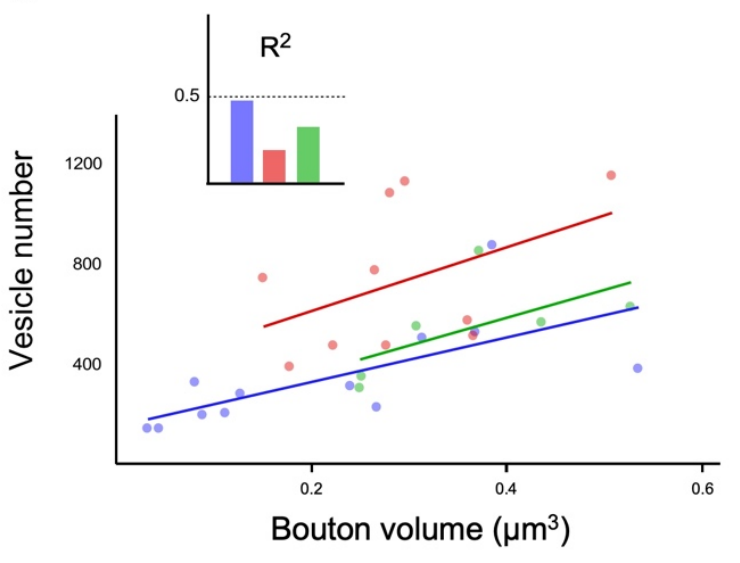

1042 Supplementary Fig. SM5. Correlation analyses between structural parameters of Po

1043 synaptic boutons in the three neuropil regions analyzed. (a-d) Dot plots showing the

1044 correlations between: (a) bouton volume vs. mitochondrial volume; (b) PSD surface

1045 area vs. spine head volume; (c) bouton volume vs. PSD surface area; and (d) bouton

1046 volume vs. number of synaptic vesicles. Correlations are indicated by linear regression

1047 as well as by the coefficient of determination ( $\mathrm{R}^{2}$, bar histograms). 
a Data plot from S1-L1 Po boutons in
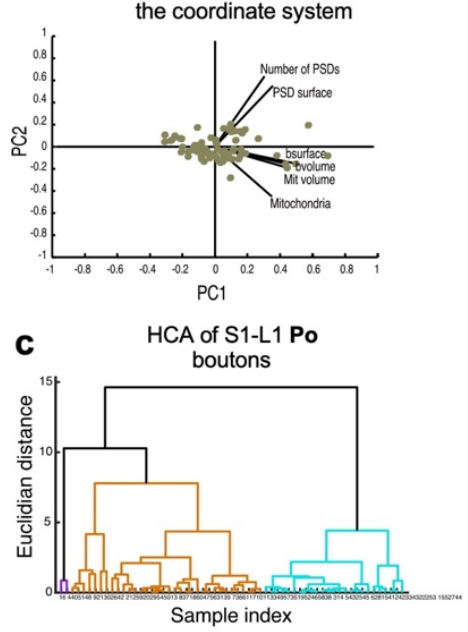

e HCA of MC-L4/3 Po

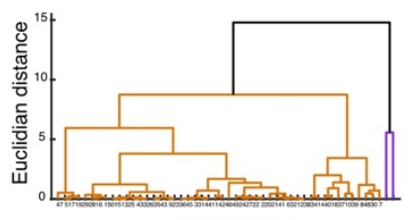

Sample index
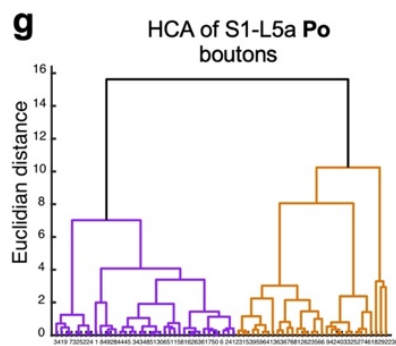

Sample index

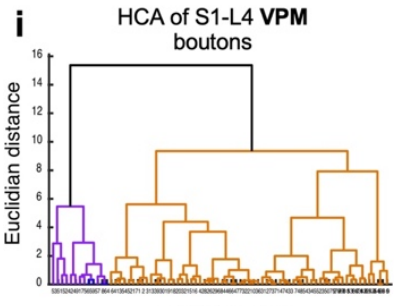

Sample index

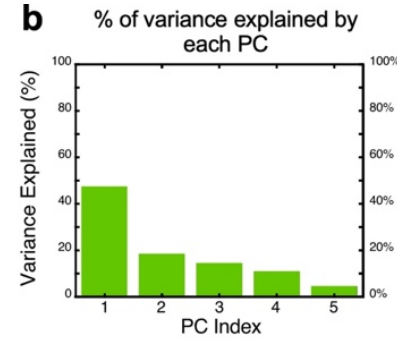

d Scatter plot of S1-L1 Po
boutons

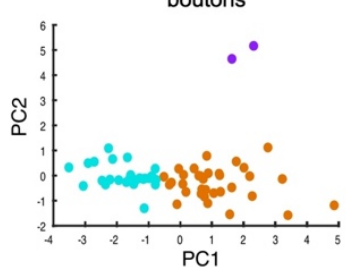

f Scatter plot of MC-L4/3 Po

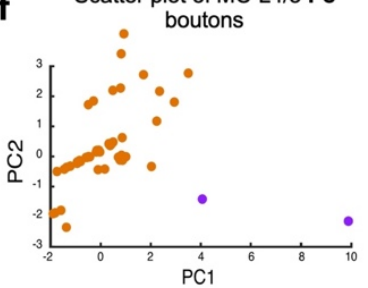

h
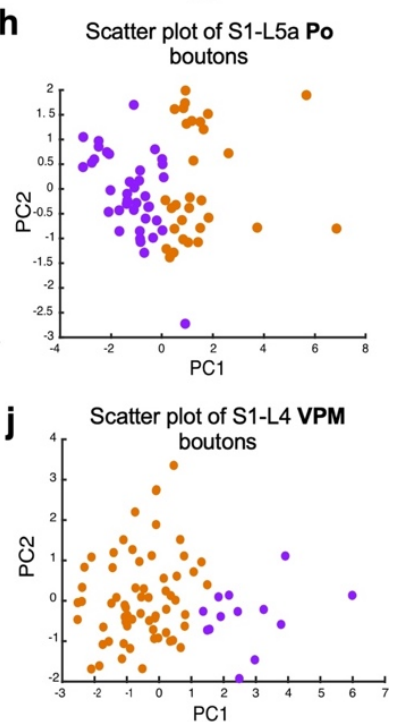

1051 Supplementary Fig. SM6. Cluster Analysis. (a) Projection of the original data of S1-

1052 L1 Po synaptic boutons in the new coordinate system based on the Principal

1053 Components (PCs), showing the coefficients for each variable and scores for each

1054 observation. Abbreviations used: bvolume: bouton volume; bsurface: bouton surface area; Mit volume: mitochondrial volume. (b) Histogram of the percentage of variance explained by the PCs for data from S1-L1 Po synaptic boutons. The PCs considered as main PCs are 1 and 2 as their total explain variance is about $70 \%$. (c-j) Dendrogram and scatter plot analyses of Po synaptic bouton parameters in each of the cortical regions 
1059 examined. (c, d) Data plots for S1-L1; (e, f) Data plots for MC-L4/3; (g, h) Data plots

1060 for S1-L5a. In addition, the same type of data plots for VPM boutons in S1-L4 ${ }^{25}$ is

1061 shown in panels (i, j). In all plots, orange and purple colors are used to show the major

1062 clusters with respect to the synaptic parameters analyzed. Dissimilarity between clusters

1063 is indicated by the Euclidean distance.

1064

1065

1066 
a

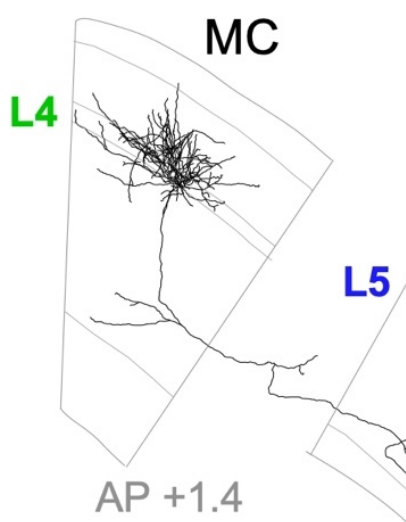

1067

1068

1069

1070

1071

1072

1073

1074

1075

1076

1077

1078

1079

1080

1081

1082 $(* *): p<0.01 ;(* * *): p<0.001$ b
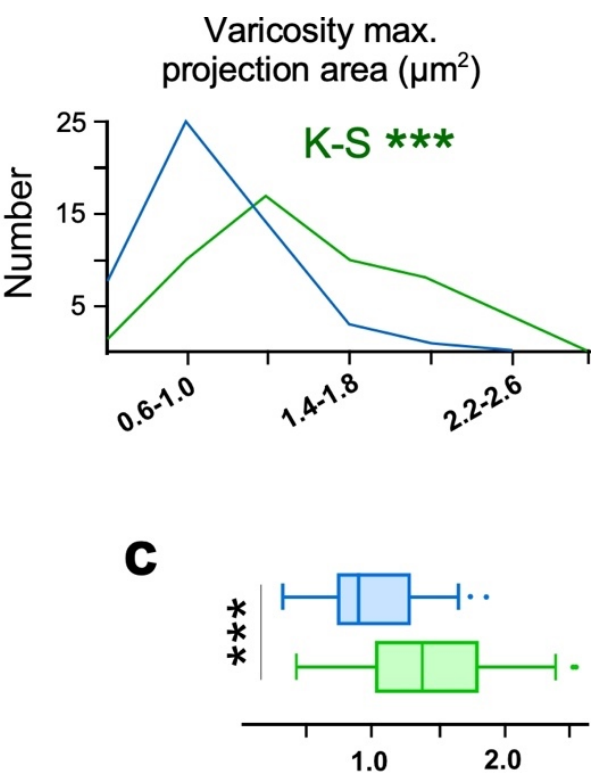

Supplementary Fig. SM7. Axon reconstruction and varicosity size analysis of a further transfection-labeled Po neuron projecting both to MC and S1. (a) Camera lucida reconstruction of the axonal arborizations and projection pattern of an individuallylabeled Po neuron targeting both MC and S1. For anatomical reference, outline sketches of cortical layers (grey lines) are shown as a background and the approximate distance to bregma (rostral "+” or caudal “-“, in $\mathrm{mm}$ ) is indicated. The black arrow indicates the point of entry in the cortical hemisphere of the axon coming from the thalamus. Scale bar represents $500 \mu \mathrm{m}$ (b) Comparison of varicosity sizes distributions (maximal projection areas) of the MC L4/3 vs. S1-L5a branches of the Po axon. This particular cell had virtually no axon branches in S1-L1. K-S = Kolmogorov-Smirnov; (c) Comparison of mean maximal projection area. Mann-Whitney U Test. (*): $p<0.05$; 
1083 Supplementary video SMV1. Video showing an example of thalamocortical Po axonal 1084 segments 3D-reconstructed in S1-L5a from FIB-SEM image stacks.

1085

1086 Supplementary video SMV2. Video showing an example of thalamocortical Po axonal 1087 segments 3D-reconstructed in S1-L5a from FIB-SEM image stacks.

1088

1089 These videos will be available will be made available before publication in the Human

1090 Brain project Graph Data Platform https://www.humanbrainproject.eu/explore-the-

1091 brain/search" under a CC-BY license.

1092

1093 